\title{
Composition and Topographic Organization of Signals Sent From the Frontal Eye Field to the Superior Colliculus
}

\author{
MARC A. SOMMER AND ROBERT H. WURTZ \\ Laboratory of Sensorimotor Research, National Eye Institute, National Institutes of Health, Bethesda, Maryland 20892-4435
}

Sommer, Marc A. and Robert H. Wurtz. Composition and topographic organization of signals sent from the frontal eye field to the superior colliculus. J. Neurophysiol. 83: 1979-2001, 2000. The frontal eye field (FEF) and superior colliculus (SC) contribute to saccadic eye movement generation, and much of the FEF's oculomotor influence may be mediated through the SC. The present study examined the composition and topographic organization of signals flowing from FEF to SC by recording from FEF neurons that were antidromically activated from rostral or caudal SC. The first and most general result was that, in a sample of 88 corticotectal neurons, the types of signals relayed from FEF to SC were highly diverse, reflecting the general population of signals within FEF rather than any specific subset of signals. Second, many neurons projecting from FEF to SC carried signals thought to reflect cognitive operations, namely tonic discharges during the delay period of a delayed-saccade task (delay signals), elevated discharges during the gap period of a gap task (gap increase signals), or both. Third, FEF neurons discharging during fixation were found to project to the SC, although they did not project preferentially to rostral SC, where similar fixation neurons are found. Neurons that did project preferentially to the rostral SC were those with foveal visual responses and those pausing during the gap period of the gap task. Many of the latter neurons also had foveal visual responses, presaccadic pauses in activity, and postsaccadic increases in activity. These two types of rostral-projecting neurons therefore may contribute to the activity of rostral SC fixation neurons. Fourth, conduction velocity was used as an indicator of cell size to correct for sampling bias. The outcome of this correction procedure suggested that among the most prevalent neurons in the FEF corticotectal population are those carrying putative cognitive-related signals, i.e., delay and gap increase signals, and among the least prevalent are those carrying presaccadic burst discharges but lacking peripheral visual responses. Fifth, corticotectal neurons carrying various signals were biased topographically across the FEF. Neurons with peripheral visual responses but lacking presaccadic burst discharges were biased laterally, neurons with presaccadic burst discharges but lacking peripheral visual responses were biased medially, and neurons carrying delay or gap increase signals were biased dorsally. Finally, corticotectal neurons were distributed within the FEF as a function of their visual or movement field eccentricity and projected to the SC such that eccentricity maps in both structures were closely aligned. We conclude that the FEF most likely influences the activity of SC neurons continuously from the start of fixation, through visual analysis and cognitive manipulations, until a saccade is generated and fixation begins anew. Furthermore, the projection from FEF to SC is highly topographically organized in terms of function at both its source and its termination.

The costs of publication of this article were defrayed in part by the payment of page charges. The article must therefore be hereby marked "advertisement" in accordance with 18 U.S.C. Section 1734 solely to indicate this fact.

\section{N T R O D U C T I O N}

The importance of the frontal eye field (FEF) and the superior colliculus (SC) to oculomotor behavior is well established. Electrical stimulation using low currents in either structure elicits saccadic eye movements (Bruce et al. 1985; Robinson 1972; Robinson and Fuchs 1969; Schiller and Stryker 1972) or inhibits them such that the eyes stay fixed (Burman and Bruce 1997; Munoz and Wurtz 1993b). Neurons in both FEF and SC discharge immediately before saccade initiation (Bruce and Goldberg 1985; Schiller and Koerner 1971; Wurtz and Goldberg 1971) or during fixation (Bizzi 1968; Munoz and Wurtz 1993a; Suzuki et al. 1979). Reversible inactivation of either structure severely disrupts saccades and fixations (Dias and Segraves 1999; Hikosaka and Wurtz 1985, 1986; Schiller et al. 1987; Sommer and Tehovnik 1997). Ablation of either structure impairs saccades and fixations for a few days or weeks, but animals then recover and exhibit only a few types of long-term deficits (e.g., Deng et al. 1986; Schiller et al. 1987). If both the FEF and the SC are bilaterally ablated, however, the ability to make saccades and fixations is permanently devastated (Schiller et al. 1980).

The FEF projects to the SC (reviewed by Leichnetz and Goldberg 1988), and there is evidence that much of the FEF's influence over oculomotor behavior is mediated by this projection. The FEF relays oculomotor signals to the SC (monkey: Segraves and Goldberg 1987; cat: Weyand and Gafka 1998b), electrical stimulation of FEF modulates neuronal activity in the SC (monkey: Schlag-Rey et al. 1992; cat: Guitton and Mandl 1974), and if the SC is reversibly inactivated, the ability to evoke saccades electrically from the FEF is impaired (Hanes and Wurtz 1999).

To understand the role of the FEF in the oculomotor system, therefore, it is important to elucidate the nature of the signals relayed from FEF to SC. One way to accomplish this is to record from neurons in the FEF that are identified as projecting to the SC by virtue of their antidromic activation after stimulation of the SC. In a landmark application of this method, Segraves and Goldberg (1987) concluded that the output of FEF to the SC primarily consists of signals related to saccade generation or suppression. Because the FEF is known to contain a wide variety of signals, from purely visual to purely motor in nature (Bruce and Goldberg 1985; Schall 1991), the FEF's output to SC appeared to be "selectively enriched" in motor-related signals (Goldberg and Segraves 1990; Segraves and Goldberg 1987).

When we began the present study, no one had examined the projection from FEF to SC in the monkey since Segraves and Goldberg (1987). Of necessity, therefore our first goal was to 
determine the composition of signals in this projection so as to replicate the previous findings. To characterize the signals flowing from FEF to SC as carefully as possible, we took advantage of techniques that have become common in the past decade: 1) use of a grid system for aiming electrodes that facilitates the systematic exploration of a cortical region and 2) statistical analysis of spike trains that facilitates the objective classification of signals carried by neurons. Also, we tried to correct for sampling bias caused by cell size variation using a method adapted from primary motor cortex research.

The present study was primarily motivated by three recent findings regarding SC neurons. First, there are tonic neuronal discharges in the SC that intervene after the phasic response to a visual target for a saccade and before the phasic discharge that is synchronized to saccade initiation. These tonic delay signals can predict hundreds of milliseconds in advance where, or whether, a saccade will be made, suggesting that they help to mediate attention, memory, or planning (Basso and Wurtz 1998; Glimcher and Sparks 1992; Kojima et al. 1996; Munoz and Wurtz 1995; Sommer et al. 1997). Second, neuronal discharges in the SC can increase during a temporal gap that elapses after a foveated spot disappears and before a visual target for a saccade appears in the periphery (Dorris and Munoz 1998; Munoz and Wurtz 1995); such gap signals are correlated with relatively fast reaction times (Saslow 1967) and therefore may help mediate fixation disengagement or movement planning (e.g., Paré and Munoz 1996; Reuter-Lorenz et al. 1995). Third, neurons concentrated in the rostral pole of the monkey SC exhibit tonic discharges during steady fixation (Munoz and Wurtz 1993a). Often, neurons with these fixation signals also pause during saccades and show an increase in activity after saccades, all of which suggests that the neurons are important for keeping the eyes still. All three of these recently studied types of signals in the $\mathrm{SC}$ also have been found in the FEF (Bizzi 1968; Bruce and Goldberg 1985; Dias and Bruce 1994; Funahashi et al. 1989; Hanes et al. 1998; Joseph and Barone 1987).

The second goal of this study, therefore, was to examine the properties of delay, gap, and fixation signals that may be relayed from FEF to SC. The report of Segraves and Goldberg (1987) did not discuss delay and gap signals. Both types of signals are thought to help mediate cognitive operations, as noted above, and if neurons projecting from FEF to SC were found to carry these signals, this would reveal a distinct subcortical route through which frontal lobe cognitive operations might influence oculomotor behavior. Segraves and Goldberg (1987) previously demonstrated that fixation-related signals are relayed from FEF to SC, and they briefly commented that FEF neurons with such signals could be activated from "a wide range of points" on the SC map (their p. 1399). We followed up on this note by systematically testing whether fixationrelated signals might project preferentially to the rostral as opposed to the caudal SC. If a rostral bias were present, this would provide strong evidence that the FEF is a source of the fixation-related signals carried by rostral SC neurons.

Our third goal was to analyze the topography of visual and movement fields in the FEF and determine how this topography projects onto the map of space within the SC. It has long been known that visual and movement space is represented topographically across the SC; in particular, the eccentricities of visual receptive fields and movement fields decrease grad- ually from caudal to rostral (Apter 1945; Cynader and Berman 1972; Sparks et al. 1976). In the FEF, the eccentricities of visual and movement fields appear to decrease from mediodorsal to ventrolateral (Bruce et al. 1985). Three important aspects of the FEF eccentricity gradient, however, are still unknown. First, it is not known which laminae in the FEF contain this eccentricity map. We examined whether an eccentricity map exists specifically in the corticotectal population of FEF neurons concentrated in layer V (Fries 1984; Leichnetz et al. 1981). Second, the exact angle of the eccentricity gradient across the two-dimensional area of the FEF is unknown. To explicitly determine this angle, we plotted eccentricity as a function of two-dimensional location in the FEF. Third, it is unknown whether the FEF's eccentricity gradient projects directly onto the known gradient of eccentricity in the SC. We analyzed this by comparing the eccentricities represented by FEF neurons to the collicular termination zones of these neurons. Prior, less direct evidence for a superposition of FEF and SC eccentricity maps has come from a variety of anatomic (Komatsu and Suzuki 1985; Stanton et al. 1988) and electrophysiological studies (Schlag-Rey et al. 1992; Segraves and Goldberg 1987).

In the present study, we first physiologically identified the FEF and SC (Fig. 1A) and implanted stimulating electrodes in the rostral and caudal SC (Fig. 1B). We then characterized the task-related signals of FEF neurons that were antidromically activated from the SC (Fig. 1C), estimated the locations of these neurons within the FEF, and estimated their termination locations along the rostrocaudal axis of the SC. We found that essentially all the neuronal types previously identified in the FEF can be antidromically activated from the SC, suggesting that the output of the FEF to the SC is not selective but reflects the general population of FEF signals. A large proportion of FEF corticotectal neurons exhibited delay signals, gap signals, or both. Surprisingly, FEF neurons with activity strongly related to the act of fixating did not project preferentially to rostral SC, although other types of corticotectal neurons did. We found a two-dimensional map of eccentricity in the FEF corticotectal neuron population and showed that it projects in an orderly manner onto the map within the SC.

Brief reports pertaining to some of these data have appeared previously (Sommer and Wurtz 1998a,b, 1999; Wurtz and Sommer 1998).

\section{METHODS}

\section{Surgery}

Two monkeys (Macaca mulatta) were surgically prepared in aseptic conditions using isofluorothane anesthesia. We inserted eye coils subconjunctivally (Judge et al. 1980), drilled and tapped holes in the skull for the placement of screws, and trephined holes for accessing the FEF (this hole was centered at A25, L20 for monkey $H$ and at A23, L18 for monkey $C$ ) and the SC (this hole was centered on the midline and angled $42^{\circ}$ back from vertical so that electrode penetrations would approach the SC approximately orthogonally to its surface, for both monkeys). Recording chambers were placed over the trephinations, plugs were attached for accessing eye coil leads, and dental acrylic was applied so that the cylinders, eye coil plugs, and a post for head restraint all were held securely, and so that the entire implant was connected firmly to the skull via the screws. To permit magnetic resonance images (MRIs) of the monkeys, screws were titanium and the chambers and head holder were plastic. Monkeys received anal- 
A

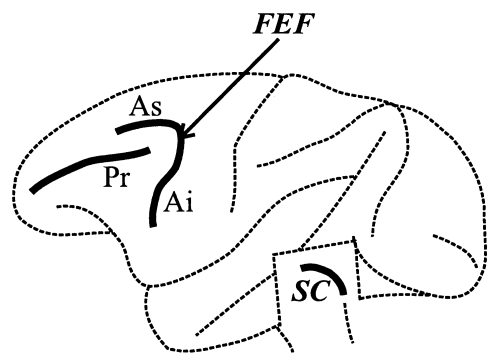

B

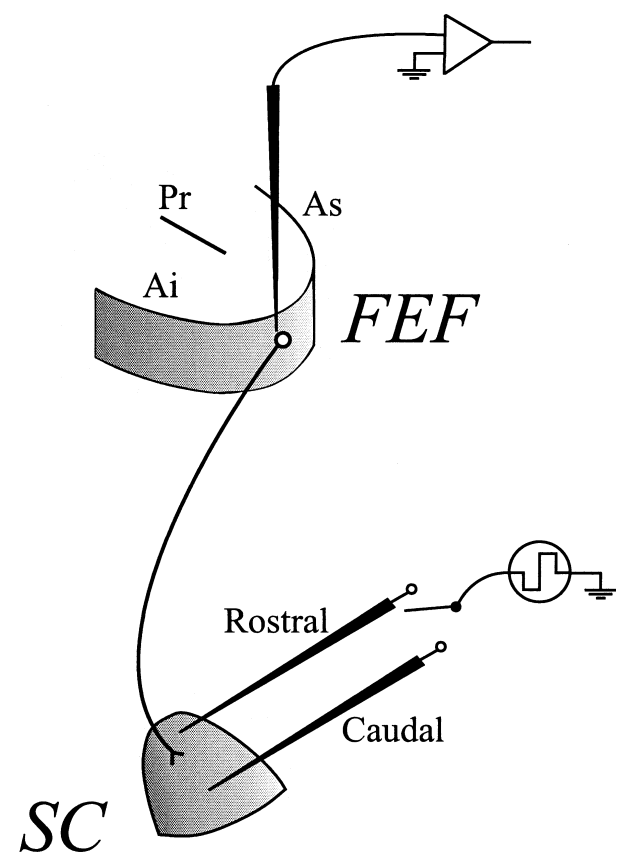

C

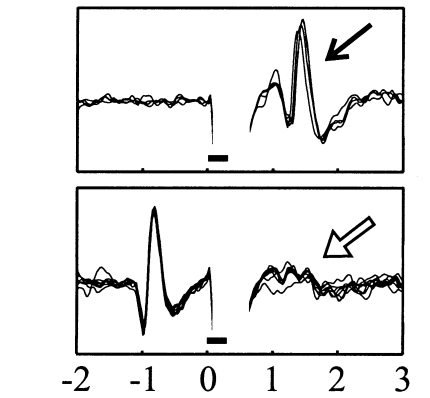

Time re: SC Stimulation (ms)

FIG. 1. Techniques used for studying signals sent from frontal eye field (FEF) to superior colliculus (SC). A: lateral view of the macaque brain is shown, with brain stem revealed in a cutaway view. FEF and SC are labeled. For reference in subsequent figures, the principal sulcus $(\operatorname{Pr})$ and the arcuate sulcus's superior (As) and inferior (Ai) branches are indicated. $B$ : the FEF, in the rostral bank of the arcuate sulcus, is depicted as if removed from the rest of the brain. We isolated and studied single neurons in the FEF that were activated antidromically from electrodes implanted in the rostral and the caudal SC. $C$ : action potential waveforms are shown to illustrate application of the collision test. Top: a single biphasic pulse of electrical stimulation in the SC (beginning at time 0 and lasting for $0.3 \mathrm{~ms}$ as indicated by solid bar) reliably caused the neuron, recorded in FEF, to fire at $1.0 \mathrm{~ms}$ latency (black arrow). Stimulus artifact is erased for clarity. Bottom: if stimulation in the SC was synchronized to occur $\leq 1.4 \mathrm{~ms}$ after the FEF neuron fired spontaneously, then stimulation failed to evoke an action potential in the FEF neuron (white arrow). The spontaneous spike traveling away from the cell body collided with and annihilated the stimulation-evoked spike traveling toward the cell body. This neuron therefore passed the collision test, demonstrating that it projected to the SC. gesics and antibiotics postoperatively. All procedures were approved by the Institute Animal Care and Use Committee and complied with Public Health Service Policy on the humane care and use of laboratory animals.

\section{Antidromic stimulation}

The FEF and the SC (Fig. 1A) were located physiologically. To find the FEF, we explored the cortex rostral to the arcuate sulcus (the sulcus was visible through the dura mater during surgery). We defined the mediolateral range of FEF in our monkeys as those sites just rostral to the arcuate sulcus where penetrations yielded saccaderelated corticotectal neurons (Visuomovement or Movement Neurons as defined in RESULTS; see Fig. 7, $A$ and $D$ ). We verified that these sites were within the FEF of Bruce et al. (1985) by electrically evoking saccades from these sites, or immediately adjacent sites, at low current threshold ( $<50 \mu \mathrm{A}$ using $70-\mathrm{ms}$ trains of biphasic pulses, $0.25 \mathrm{~ms} /$ phase, at $350 \mathrm{~Hz}$ ). Threshold was defined as current that evoked saccades on $50 \%$ of trials. Stimulation began $200 \mathrm{~ms}$ after disappearance of a foveated light while the monkey's task was to maintain fixation on the blank screen (during the fixation task described in Behavioral procedures). The SC was identified physiologically by its characteristic lamination, with visually responsive neurons located dorsal to neurons discharging before and during saccade generation (e.g., Sparks and Hartwich-Young 1989), and by its topographic map of stimulation-evoked saccades (Robinson 1972).

Stimulating electrodes were implanted in rostral and caudal SC (Fig. $1 B$ ). They were used for 1-3 mo and then replaced at slightly different locations once they began to fail (i.e., when their ability to conduct current degraded). Electrodes in rostral $S C$ all were within the $3^{\circ}$ amplitude representation on the SC map (Robinson 1972), and those in caudal $S C$ all were between the 7 and $20^{\circ}$ amplitude representation. Electrodes were placed near the representation of the horizontal meridian. We chose the depth of stimulation according to the following criteria. Rostral SC electrode tips were placed at depths in the SC where visual receptive fields were foveal, where activity continued while the monkey fixated a spot that blinked off for several hundred milliseconds, and where we could delay ipsiversive visually guided saccades, evoke small contraversive saccades, or both, using $<20 \mu \mathrm{A}$. In practice, these criteria led to placement of rostral electrode tips $2.4 \pm 0.6(\mathrm{SD}) \mathrm{mm}$ below the SC surface (range 1.6-3.3 $\mathrm{mm}$ ), where saccades were inhibited or $<3^{\circ}$ amplitude saccades were evoked at thresholds of $9.5 \pm 5.1 \mu \mathrm{A}$ (range 3-14 $\mu \mathrm{A})$. When placing caudal SC electrode tips, we chose sites where large contraversive saccades were evoked using $<10 \mu \mathrm{A}$ and where the dominant neuronal discharge was presaccadic burst activity. These criteria led to placement of caudal electrode tips $1.8 \pm 0.5 \mathrm{~mm}$ below the SC surface (range 1.5-2.7 mm) where saccades of amplitude $12 \pm 6^{\circ}$ (range $7-20^{\circ}$ ) were evoked using current thresholds of $4.3 \pm 1.9 \mu \mathrm{A}$ (range 2-7 $\mu \mathrm{A}$ ). Distances between the rostral and caudal electrode tips in the SC, as estimated from inter-electrode distances in the grid, were $1.8 \pm 0.4 \mathrm{~mm}$ (range 1.4-2.2 mm). The characteristics of the rostral SC stimulation sites indicated that they were in the intermediate gray layer zone where "fixation" neurons are found (Munoz and Wurtz 1993a), and the characteristics of caudal sites indicated that they were in the intermediate gray layer region where saccade-related "burst" and "buildup" neurons are found (Munoz and Wurtz 1995).

Once the SC stimulating electrodes were cemented into place (by applying epoxy to bind together the electrode shafts, guide tubes, grid, and implanted cylinder), near daily recording sessions commenced. During a penetration through the FEF, we first isolated a neuron and then attempted to activate it from the rostral or the caudal SC using a single biphasic pulse of current (cathodal-anodal, $0.15 \mathrm{~ms}$ and $600 \mu \mathrm{A}$ per phase). Once an activated neuron was found we lowered the current to find the threshold for activating it from each electrode (threshold defined as current for which activation occurred $50 \%$ of the time). Antidromic activation was confirmed for all neurons in this 
study using the collision test (Fig. 1C) (see Lemon 1984 for review of the collision test). Activation latency was measured from the start of the stimulation artifact until the start of the evoked action potential. Conduction velocity was calculated using the formula $D /(L-u)$, where $D$ is the axon distance from FEF to SC (estimated to be 40.5 $\mathrm{mm}$ ) (Segraves and Goldberg 1987), $L$ is the antidromic activation latency, and $u$ is the utilization time, i.e., the time it takes for electrical stimulation to elicit an action potential, estimated to be $0.2 \mathrm{~ms}$ (reviewed by Lemon 1984).

We used tungsten microelectrodes (Frederick Haer) for recording and stimulating (impedances were $300-1,200 \mathrm{k} \Omega$ and $90-110 \mathrm{k} \Omega$ at $1,000 \mathrm{~Hz}$, respectively). Electrodes were inserted through a guide tube held by a grid (Crist et al. 1988) that was attached within the implanted chamber. Use of the grid was especially important for recordings, because it permitted systematic exploration of the cortex within and surrounding the FEF. For the FEF, guide tubes were made as short as possible, so that they barely passed through the dura (to avoid damaging the cortex). For the SC, guide tubes were inserted so their ends were $\sim 4 \mathrm{~mm}$ above the SC surface.

\section{Behavioral procedures}

During experimental sessions, a monkey sat in a primate chair centered within magnetic fields used for detecting eye position (Robinson 1963). Visual stimuli $\left(0.3 \times 0.3^{\circ}\right.$ blue or red spots on a dark background) were back-projected onto a tangent screen $57 \mathrm{~cm}$ in front of the monkey using an LCD projector (Sharp model 850). Ambient room light was dim. Coverage of the visual field was $80^{\circ}$ horizontally and $60^{\circ}$ vertically, centered on a point straight ahead from the midpoint of the monkey's eyes. A personal computer controlled the presentation of visual stimuli, and this computer in turn was controlled by a personal computer running a QNX-based real time experimentation data acquisition system (REX) (Hays et al. 1982). A third personal computer ran in-house software that served as a digital oscilloscope $(50 \mathrm{kHz})$, allowing us to separate action potential waveforms using time and amplitude windows. The REX system recorded at $1 \mathrm{kHz}$ the eye position, the occurrence of action potentials, and the timing of task events. Visual stimuli actually appeared on the screen an average of $12 \mathrm{~ms}$ after the times noted in data files, as reported previously (Basso and Wurtz 1998; Eifuku and Wurtz 1998), and we accounted for this by shifting these times in the data files later by $12 \mathrm{~ms}$.

Monkeys performed three tasks that allowed us to characterize the signals carried by neurons: the delayed-saccade task, the gap task, and the fixation task.

DELAYED-SACCADE TASK. At the beginning of the delayed-saccade task (Fig. 2A), a monkey was required to fixate a central spot of light for a random duration $(500-800 \mathrm{~ms})$, and then a target appeared in the periphery. In visual trials (Vis. in Fig. $2 A$ ), the target remained lit for the rest of the trial; in memory trials (Mem. in Fig. 2A), the target disappeared after $100 \mathrm{~ms}$. We randomly interleaved these two versions of the delayed-saccade task to help us identify tonic visual responses, as described in RESULTS. After a random delay period of $500-1,000 \mathrm{~ms}$, the fixation light disappeared, providing the cue to make a saccade to the target's location. When analyzing results, the mean firing rates during five periods (Analysis Epochs in Fig. 2A) were quantified and compared (statistical analysis is described in STATISTICAL ANALYSIS OF NEURONAL SIGNALS). The Baseline epoch was 500-200 ms before target onset, the Visual epoch was $50-150 \mathrm{~ms}$ after target onset, the Delay Period epoch was 300-0 ms before the cue to move, the Presaccadic epoch was 50-0 ms before saccade initiation, and the Postsaccadic epoch was 50-150 ms after saccade termination.

GAP TASK. In the gap task (Fig. 3A), the monkey first fixated a spot for a random duration of 500-800 ms, and then the spot disappeared. The monkey had to maintain fixation on the blank screen and then, after a 200-ms gap period, a target was presented; the monkey could then look at the target with no further imposed delay. Firing rate during a Gap Period epoch, from $50 \mathrm{~ms}$ before target onset to $50 \mathrm{~ms}$ after, was compared with firing rate during a Baseline epoch 500-200 ms before start of the gap. We inspected all the data to ensure that the Gap Period epoch did not overlap with periods of phasic peripheral visual activity occurring after target onset.

FIXATION TASK. In the fixation task (Fig. 4A), the monkey foveated a spot for a random duration of $500-1,000 \mathrm{~ms}$, and then the spot disappeared for a random duration of 400-600 ms while the monkey had to maintain fixation. Then the spot reappeared at the same place for an additional random duration of 500-1,000 ms. Five analysis epochs were defined. The Baseline epoch was during the intertrial period, 300-0 ms before fixation spot onset, the First Fixation epoch was 100-300 ms after start of fixation, the Foveal Visual Offset epoch was 100-300 ms after disappearance of the fixation spot, the Second Fixation Epoch was 300-0 ms before fixation spot reappearance, and the Foveal Visual Onset epoch was 100-300 ms after fixation spot reappearance. Firing rates during all five epochs were quantified and compared with each other statistically, as described below, but only a few of the resulting comparisons were found to be useful for characterizing the neurons, as described in RESULTS.

GENERAL TESTING PROCEDURE. The basic protocol for characterizing the signals carried by each antidromically activated neuron was as follows. First, we determined the extent of the neuron's visual receptive field or movement field by having the monkey perform the delayed-saccade or gap task while the position of the target was varied throughout the testing space until a location was found that evoked maximal visual- or movement-related firing (as judged by on-line inspection of action potential rasters and histograms). This location was termed the best location for the neuron. Then, during formal testing using the delayed-saccade and gap task, the visual target was presented at this best location (for a minority of neurons, visual- and movement-related activity did not vary throughout the testing space, i.e., there was no best location, so during formal testing of these neurons we arbitrarily chose to present the target contralaterally, $10^{\circ}$ eccentric on the horizontal meridian). Often a separate block of gap task trials also was run in which the target location was randomized twofold (described in RESULTS), to see whether gap-related signals depended on knowledge of eventual target location and to help facilitate comparison of our results with those of Dias and Bruce (1994). If a neuron appeared to change its firing rate at the start of fixation during the delayed-saccade or gap tasks, the monkey was then run on the fixation task to better characterize the foveal-related signals. Eye position tolerance windows around fixation spots were $2 \times 2^{\circ}$, and those around target stimuli typically were $5 \times 5^{\circ}$ (sometimes larger for targets in the far periphery). Correct responses were rewarded with drops of water during experiments (water intake was controlled in the monkey's home cage).

STATISTICAL ANALYSIS OF NEURONAL SIGNALS. For each neuron and each type of task, the data set consisted of mean firing rates during the series of epochs associated with the task events. First, to see whether the neuron's activity varied at all during the task, we ran an ANOVA on the data. If this was significant $(P<0.01)$, we then performed an all-pairwise multiple comparison test (Student-Newman Keuls or Dunn's) so that we could determine whether firing rates in any two epochs differed from each other $(P<0.05)$. Specific types of signals (e.g., delay signals) were defined according to comparisons between epochs, as presented in RESULTS.

\section{Estimating cell body and axon termination locations}

To estimate the location of a neuron's soma in the FEF, we moved the recording electrode carefully up and down until the action potential voltage was peak-to-peak maximal and then recorded the depth of the electrode tip with respect to the end of the guide tube. Over the 
A Delayed-saccade Task

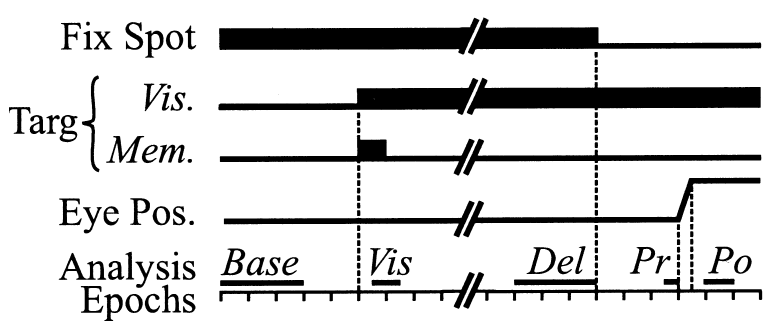

B Neuron with delay signal

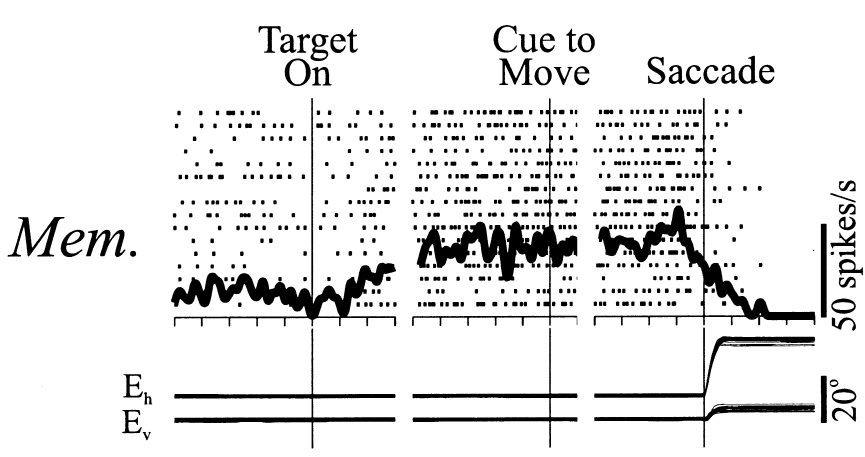

C Visual Neurons

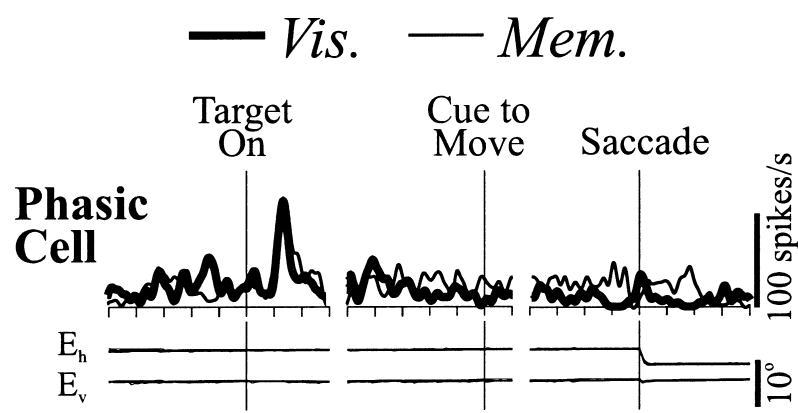

D Movement Neuron

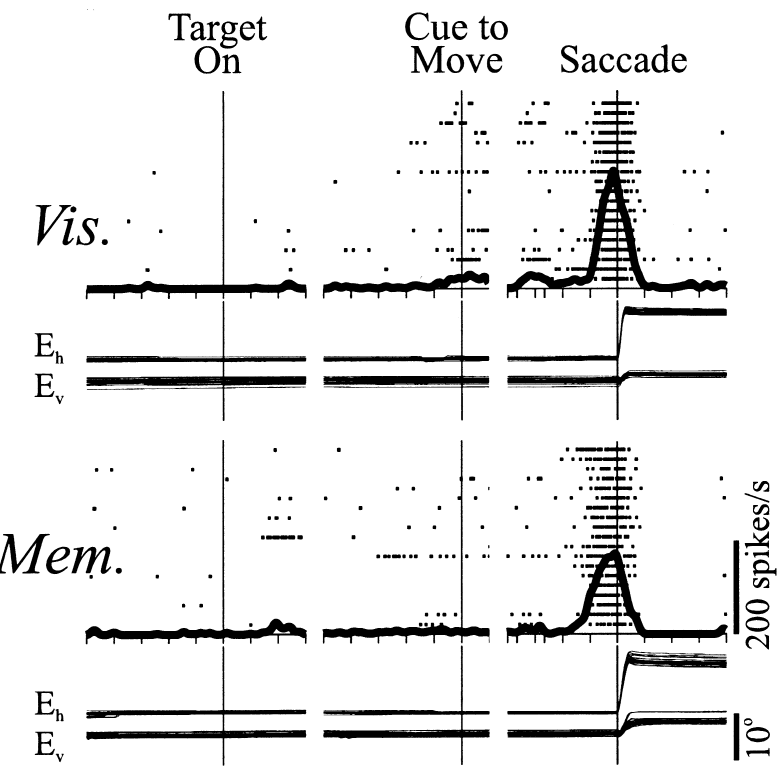

\section{E Visuomovement Neuron}

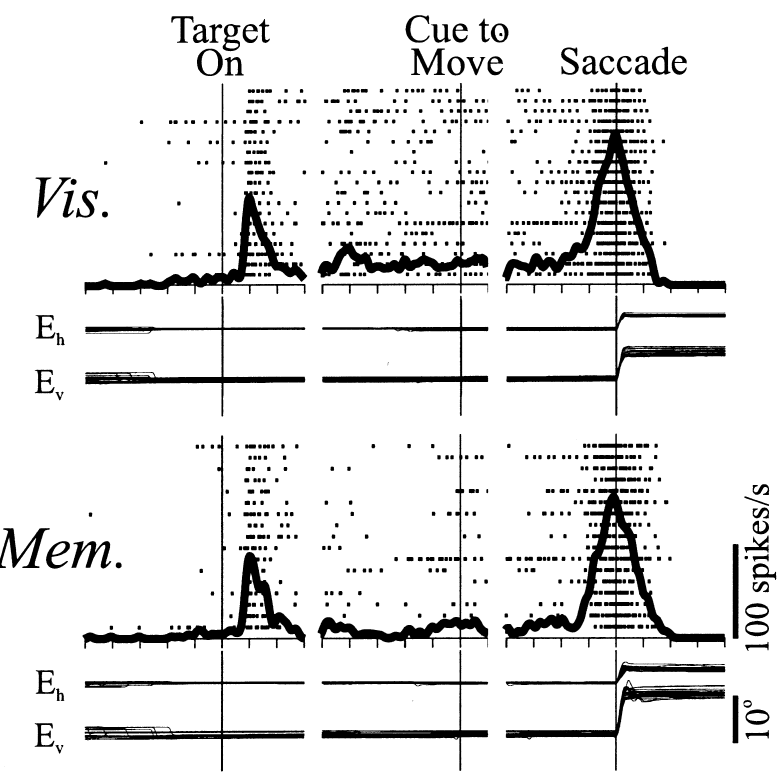

FIG. 2. Delayed-saccade task and some of the signals and neuron types identified using it. A: schematic of the task is shown. After the monkey fixated a light (Fix Spot) for a random duration, a target (Targ) appeared in the periphery. In Visual Trials (Vis.), the target remained lit for the rest of the trial; in Memory Trials (Mem.), the target disappeared after $100 \mathrm{~ms}$. The monkey was allowed to make a saccade to the target location (Eye Pos.) only after a randomized delay period elapsed. The cue to move was disappearance of the Fix Spot. Firing rates during 5 periods (Analysis Epochs) were statistically compared: Base, Baseline epoch; Vis, Visual epoch; Del, Delay Period epoch; Pr, Presaccadic epoch; Po, Postsaccadic epoch. Time scale in all panels is $100 \mathrm{~ms}$ per tic. $B$ : activity of a neuron carrying a delay signal. Only results from memory trials are shown, because only these trials were used to identify delay signals. Neuronal discharges in successive trials are represented by rasters, and the average firing rate is shown using a spike density function formed by convolving the individual rasters with a Gaussian of $\sigma=10 \mathrm{~ms}$ and summing the resultant curves. Eye traces $\left(E_{\mathrm{h}}\right.$, horizontal component; $E_{\mathrm{v}}$, vertical component) are shown below the rasters. Eye position and firing rate scales are shown at right. Rasters and spike density functions are aligned with respect to target onset (Target On), fixation spot offset (Cue to Move), and saccade initiation (Saccade). C: activity of 2 Visual Neurons. Top: a neuron carrying only a phasic peripheral visual signal. Bottom: a neuron carrying a strong tonic peripheral visual signal. Spike density functions from visual trials (thick lines) and memory trials (thin lines) are superimposed and rasters are omitted for clarity. Eye movements shown are from visual trials. $D$ and $E$ : activity of a Movement Neuron and a Visuomovement Neuron, respectively. In $D$ and $E$, data from visual trials are shown above and data from memory trials are shown below. 
A Gap Task

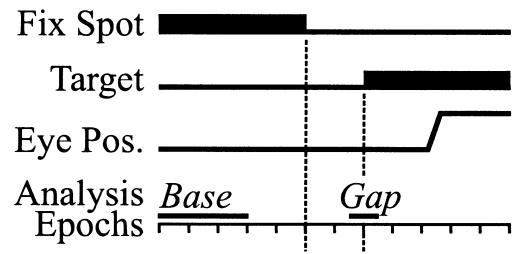

\section{B Neuron with gap increase signal}

\section{- Target in movement field Target out of movement field}

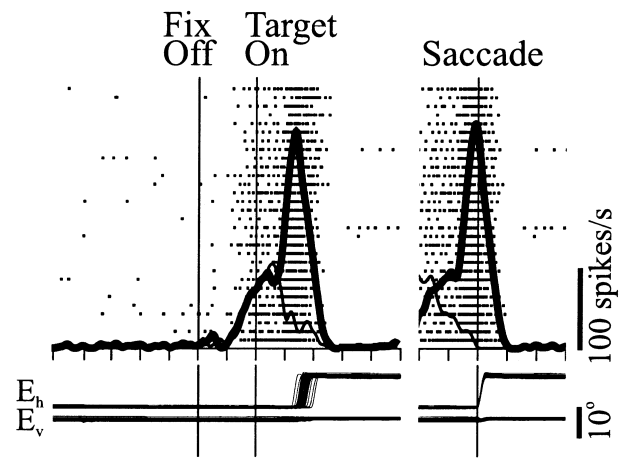

C Neuron with gap decrease signal

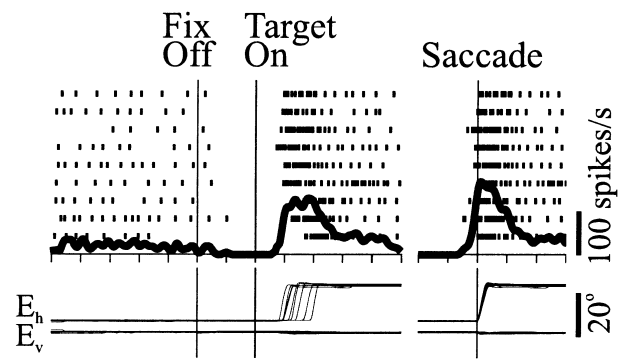

FIG. 3. Gap task and the signals identified using it. A: schematic of the task. After initial foveation of a fixation light (Fix Spot) for a random duration, the light was extinguished and fixation had to be maintained on the blank screen. After a gap period of $200 \mathrm{~ms}$, a Target appeared in the periphery. The monkey was allowed to make a saccade (Eye Pos.) as soon as the Target appeared. Analysis Epochs shown at bottom: Base, Baseline epoch; Gap, Gap Period epoch. Time scale in all panels is $100 \mathrm{~ms}$ per tic. $B$ : activity of a neuron carrying a gap increase signal. Shown are the rasters, eye movement data, and spike density function (drawn with bold line) for trials in which the target was presented in the movement field. Superimposed is the spike density function (drawn with thin line) summarizing neuronal activity when the target was presented, in randomized trials, out of the movement field; note that gap increase signals are present in both trial types, but the subsequent presaccadic burst occurs only for saccades made into the movement field. $C$ : activity of a neuron carrying a gap decrease signal. $\sigma=10 \mathrm{~ms}$ for all the spike density functions.

course of the study, after numerous penetrations, it became evident that the ends of the guide tubes for monkey $C$ rested on top of the cortex (because 1st neurons typically were encountered 0-500 $\mu \mathrm{m}$ below the end of the guide tube even though the guide tube was barely through the dura), whereas the ends of the guide tubes for monkey $H$ were $1 \mathrm{~mm}$ above the cortex. During data analysis, the noted depths of all the FEF neurons were adjusted using this corrective information to estimate how deep the neuron was located with respect to the top of the cortex. To analyze topographies within the FEF, cell body

\section{A Fixation Task}

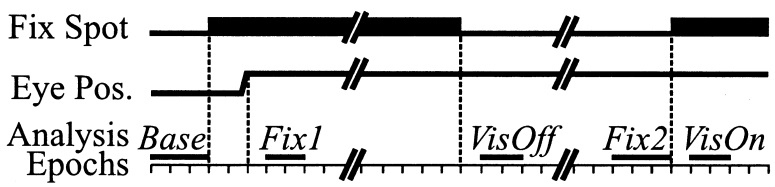

B Neuron with fix.-related signal

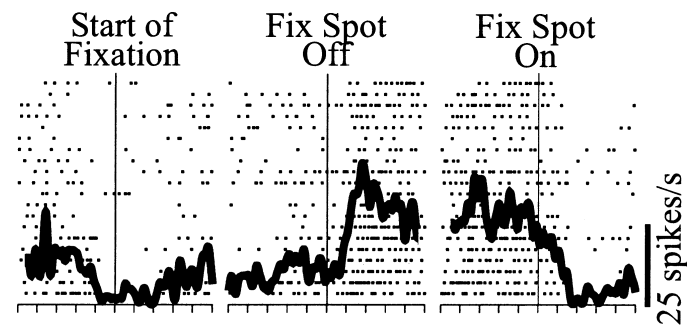

C Pure Fixation Neuron

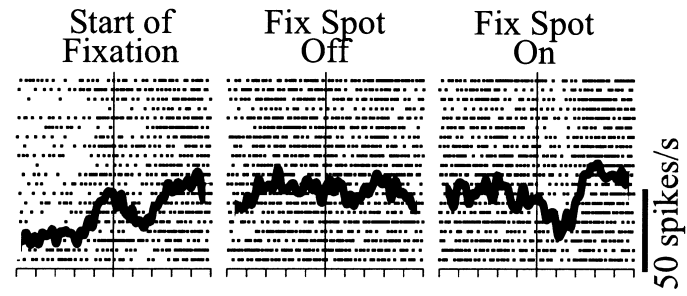

D Pure Foveal Visual Neuron

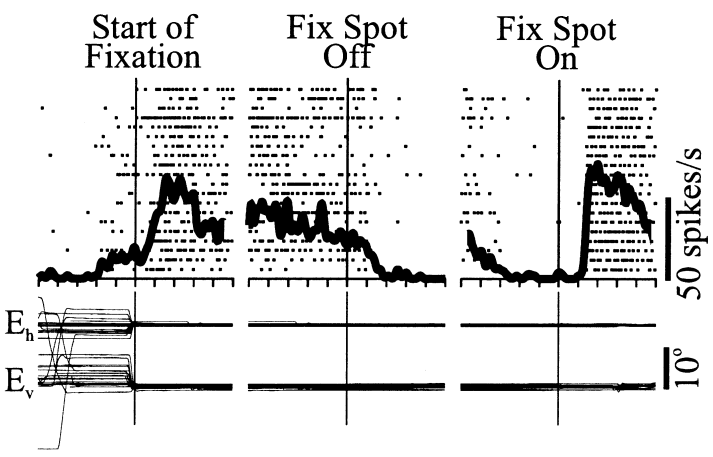

FIG. 4. Fixation task and the signals and neuron types identified using it. A: schematic of the task. A fixation spot (Fix Spot) appeared and the monkey foveated it (Eye Pos.) for a random duration. The fixation spot disappeared for $400-600 \mathrm{~ms}$ and then reappeared. Throughout the trial the monkey had to maintain steady fixation. Analysis Epochs shown at bottom: Base, Baseline epoch; Fix1, First Fixation epoch; VisOff, Foveal Visual Offset epoch; Fix2, Second Fixation epoch; VisOn, Foveal Visual Onset epoch. Time scale in all panels is $100 \mathrm{~ms}$ per tic. $B$ : activity of a neuron carrying a fixation-related signal. $C$ : activity of a Pure Fixation Neuron, a neuron that carried a fixationrelated signal and also fired fairly steadily throughout the entire fixation trial. $D$ : activity of a Pure Foveal Visual Neuron. Examples of eye positions recorded during the fixation task are shown for the data in $D . \sigma=10 \mathrm{~ms}$ for all the spike density functions. 
locations in FEF were plotted on a standard two-dimensional map [similar to the practice of plotting SC neurons on a standard map (e.g., Anderson et al. 1998)]. Derivation of the FEF standard map is described in RESULTS.

The rostrocaudal location of axon termination in the SC was estimated by comparing the current threshold, $I$, for activating each FEF neuron from the rostral versus the caudal SC electrodes. We preferred to use a quantity that increased with increasing ease of activating a neuron; therefore we defined an ability to activate, $A$, as $I^{-1}$. As examples, $A=0$ meant the neuron could not be activated antidromically from an electrode, $A=2,500$ meant that a neuron could be activated, but at relatively high current threshold $(400 \mu \mathrm{A})$, and $A=$ 100,000 meant that it could be activated very easily (using only 10 $\mu \mathrm{A})$. To compare the ability to activate a neuron at the rostral and caudal electrodes, we defined an Electrode Preference Index, EPI, using a standard contrast ratio: EPI $=\left(A_{\text {caudal }}-A_{\text {rostral }}\right) /\left(A_{\text {caudal }}+\right.$ $\left.A_{\text {rostral }}\right)$. Therefore EPI $=1$ meant that the neuron was activated only from the caudal electrode, EPI $=-1$ meant that it was activated only from the rostral electrode, and EPI $=0$ meant that it was activated with equal ease from both electrodes. These techniques appeared to provide a good estimate of where a neuron's axon terminated along the rostrocaudal axis of the $\mathrm{SC}$, as reviewed in the DISCUSSION. [Note, if one prefers to think in terms of current threshold, $I$, rather than its reciprocal, $A$, then the above equation can be rearranged to yield $\mathrm{EPI}=\left(I_{\text {rostral }}-I_{\text {caudal }}\right) /\left(I_{\text {rostral }}+I_{\text {caudal }}\right)$. $]$

\section{Correction for sampling bias}

Estimates of signal composition in a population of neurons can suffer from sampling bias due to the preferential recording of larger neurons (Towe and Harding 1970). In terms of quantities that are measurable during in vivo extracellular recordings, neurons with higher conduction velocities are oversampled (conduction velocity is directly related to cell size) (Cullheim 1978; Gasser 1941; Kernell and Zwaagstra 1981). To correct for this sampling bias we used the method described in detail by Humphrey et al. (1978) and applied to primary motor cortex data by Humphrey and Corrie (1978). Their premise is that, "with equivalent transmembrane action potentials, the discharge of a large neuron will generate a greater flow of membrane current, a larger extracellular spike, and a potential field that is above recording noise levels over a greater distance than will a small cell. Thus the larger a neuron, the greater is the distance that it may lie from an exploring electrode before its spike becomes undetectable or too small to observe reliably. Because of this relationship, the effective volume of neural tissue that is 'sampled' or 'observed' during a given microelectrode penetration is not a constant, but is instead larger when recording extracellularly from large cells than when recording from small cells. In order to estimate the true relative densities of cells of different sizes, it is necessary, therefore to divide observed measures of their relative densities or frequencies within a given sample of units by estimates of their relative, effective recording volumes $\left(V_{\text {eff }}\right)$. For example, if $N_{\mathrm{o}}(v)$ is an experimentally observed distribution of cellular conduction velocities $(v)$, then the true or unbiased distribution, $N_{\mathrm{t}}(v)$, would be given by $N_{\mathrm{t}}(\tau)=N_{\mathrm{o}}(v) / V_{\text {eff }}(v)$ where $V_{\text {eff }}(v)$ is now considered to be an explicit function of axonal conduction velocity, rather than that of the closely related quantity, cell size." (Humphrey and Corrie 1978, p. 234). Therefore the key to correcting for sampling bias due to cell size variation is to find $V_{\text {eff }}(v)$. Humphrey et al. (1978) derived an equation for pyramidal neurons that described the extracellular spike amplitude as a function of various neuronal characteristics (e.g., dendritic geometry), extracellular conductivity, and distance from the cell body's center to the recording site. Empirical measurements verified that the equation was accurate (Humphrey et al. 1978), and therefore it was used to evaluate $V_{\text {eff }}$. Further models and physiological experiments revealed a relation between extracellular spike amplitude and conduction velocity, and by combining this result with the equation noted above, Humphrey et al. (1978) concluded that $V_{\text {eff }}(v)=k v^{3 / 2}$, where $k$ is a constant.

We used the above expression for $V_{\text {eff }}(v)$ to correct for sampling bias. First, the experimentally observed distribution of conduction velocities for our sample of antidromically activated neurons was expressed as a histogram $N_{\mathrm{o}}\left(v_{\mathrm{i}}\right)$, representing the number of neurons, $N_{\mathrm{o}}$, that had conduction velocities within each bin $v_{\mathrm{i}}(18 \mathrm{bins}$ of $5 \mathrm{~m} / \mathrm{s}$
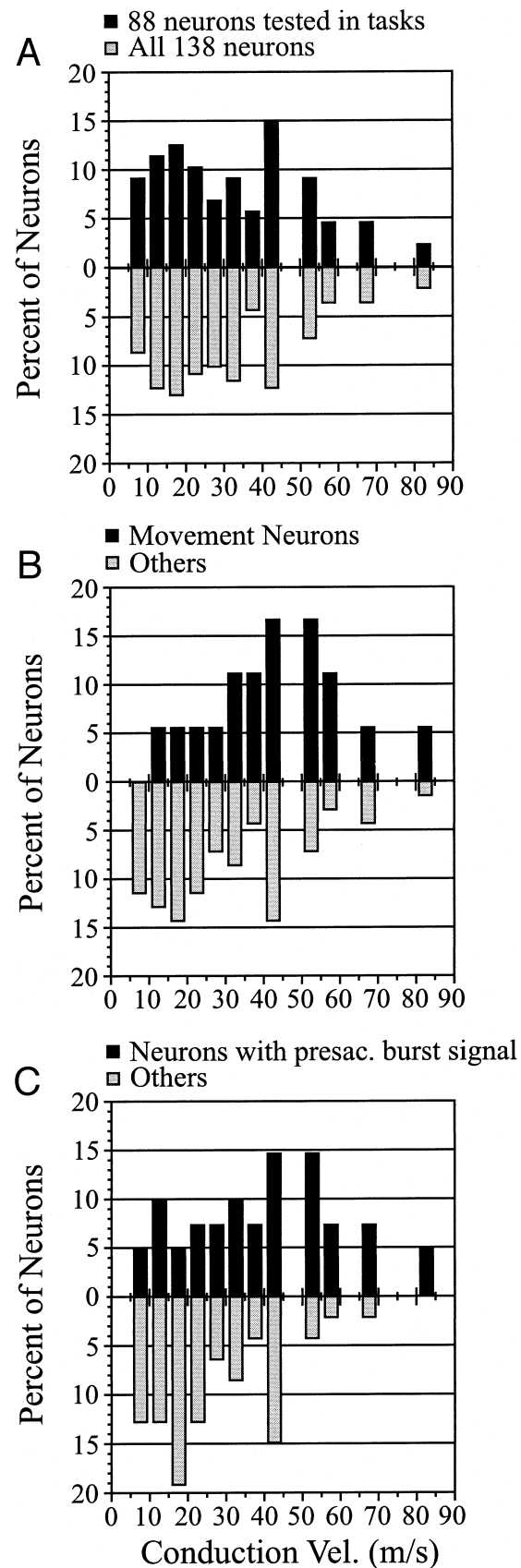

FIG. 5. Conduction velocity histograms for FEF corticotectal neurons. Graphed are the percentages of neurons in each population that had conduction velocities represented along the abscissa. To facilitate comparison between histogram pairs, in each panel one histogram is inverted and placed beneath the other. A: conduction velocity distributions for the 88 neurons tested on behavioral tasks and for the entire population of 138 neurons [note that a histogram and statistical summary of the antidromic latencies for most of these neurons $(n=133)$ was previously published (Sommer and Wurtz 1998a)]. B: conduction velocity distributions for Movement Neurons and for other neurons. $C$ : conduction velocity distributions for all neurons carrying a presaccadic burst signal and for other neurons. 
width were used, spanning 0 to $90 \mathrm{~m} / \mathrm{s}$; see Fig. 5). The estimated true distribution, therefore was $N_{\mathrm{t}}\left(v_{\mathrm{i}}\right)=N_{\mathrm{o}}\left(v_{\mathrm{i}}\right) / k v_{\mathrm{i}}^{3 / 2}$. The value of $k$ is unknown, but it can be canceled out by converting the numerical histogram, $N_{\mathrm{t}}$, into a histogram of proportions, $P$, of neurons that are in each conduction velocity bin

$$
P\left(v_{\mathrm{i}}\right)=\frac{N_{\mathrm{o}}\left(v_{\mathrm{i}}\right) v_{\mathrm{i}}^{-3 / 2}}{\sum_{\mathrm{i}=1}^{18} N_{\mathrm{o}}\left(v_{\mathrm{i}}\right) v_{\mathrm{i}}^{-3 / 2}}
$$

The value of each $v_{i}$ was set to the midpoint of each velocity bin.

In summary, this corrected distribution estimates the actual proportion of neurons in the underlying population that have various conduction velocities. The major assumption of the method is that the neurons are pyramidal such that their effective recording volumes $\left(V_{\text {eff }}\right)$ are cylindrical, aligned with the apical dendrite. Although FEF corticotectal neuron morphology is not known in detail, most neurons in FEF layer V appear to be pyramidal (e.g., Stanton et al. 1989; Walker 1940), and studies of partially filled FEF corticotectal neurons suggest that most of them are pyramidal (Fries 1984; Leichnetz et al. 1981).

Using the corrected distribution of conduction velocities, the corrected proportion of each functionally defined class of neuron can then be calculated. To illustrate this procedure, consider the simple case in which there are two conduction velocity bins, $v_{1}$ and $v_{2}$, and three neurons in the recorded sample. Assume that one neuron fell in bin $v_{1}$ and two neurons fell in bin $v_{2}$. After correction, assume that the corrected distribution indicates that $80 \%$ of neurons in the underlying population actually fall into bin $v_{1}$ and $20 \%$ fall into bin $v_{2}$. Now, assume that only one of the recorded neurons is of cell type $X$ (e.g., the class of neurons carrying delay signals) and that this neuron fell into bin $v_{2}$. In the experimentally observed data, therefore cell type $X$ made up $50 \%$ of the cells in bin $v_{2}$, or $33.3 \%$ of the entire sample. After correction, cell type $X$ still accounts for $50 \%$ of the data that fall into bin $v_{2}$, but now it has been calculated that cells with conduction velocity in bin $v_{2}$ actually make up only $20 \%$ of the underlying population. Therefore the corrected proportion of cell type $X$ in the population is $50 \%$ of $20 \%$, or $10 \%$. This procedure is easily extended to cases where there are arbitrary numbers of cell classes or conduction velocity bins. The only assumption is that neurons in the same velocity bin (i.e., neurons of similar sizes) are sampled with equal likelihood; this assumption seems valid because sampling bias appears to be caused primarily by cell size variation (Towe and Harding 1970).

\section{Anatomic verification of FEF and SC sites}

MRIs (1.5 Tesla) were taken of the brain in both monkeys, and frontal and parasagittal planes were inspected at 1-mm intervals. A few days before taking the MRI we implanted electrodes with their tips at locations in the FEF that had yielded many corticotectal neurons so that we could visualize these locations. For monkey $H$, over a series of days near the end of the experiment, we made marking lesions at the sites of antidromically activated neurons in the FEF and through the tips of the stimulating electrodes in the SC, using DC of $10 \mu \mathrm{A}$ for $20 \mathrm{~s}$ (for FEF) and $20 \mu \mathrm{A}$ for $60 \mathrm{~s}$ (for SC). About $1 \mathrm{wk}$ later we overdosed the monkey with pentobarbital sodium, inserted several guide pins into the brain through reference holes in the FEF chamber grid, and perfused the animal transcardially with $10 \%$ neutral buffered Formalin. The guide pins, fixed in place, were then used to direct blocking cuts of the FEF. We sectioned the FEF block in a plane normal to the cortical surface and parallel to the principal sulcus in $30-\mu \mathrm{m}$ sections. The SC was sectioned coronally every $30 \mu \mathrm{m}$. For both FEF and SC, in every three consecutive sections, two were stained for cell bodies (thionin) and one for myelin (modified protocol of Gallyas 1979) to aid in recovery of marking lesions. The other monkey is being used for further experiments.

\section{R E S U L T S}

We isolated 138 neurons in the FEF that were activated antidromically from the SC (monkey $H, n=82$; monkey $C, n=$ 56). Of these, 88 were analyzed using our behavioral tasks. Of the remaining 50 neurons, 4 were not modulated by any of our tasks, and 46 were lost before they could be fully tested on the tasks.

\section{Composition of signals sent from FEF to $S C$}

We analyzed neuronal discharges that occurred in relation to visual stimulation, saccade generation, fixation, delay periods, and gap periods. These discharges were the "signals" carried by neurons. Neurons that carried specific combinations of signals were grouped into "neuron types." For example, neurons discharging just after visual stimulation and also just before saccade generation were termed Visuomovement Neurons (after the nomenclature of Bruce and Goldberg 1985). Summary data for each signal type and each neuron type are listed in Tables 1 and 2, respectively.

SIGNALS RELATED TO DELAY PERIODS, PERIPHERAL VISUAL STIMULATION, OR MOVEMENT. We used the delayed-saccade task to detect signals related to delay periods, peripheral visual stimulation, and saccade generation. A neuron had a delay signal if, in memory trials, its firing rate during the delay period (Del epoch, Fig. 2A) differed from its baseline firing rate (Base epoch). Only memory trials were considered because, in the delay period and baseline epochs of these trials, visual stimulation (fixation spot on, target off) and motor behavior (steady fixation) were identical. Firing rate differences between the epochs therefore may primarily reflect cognitive processes (e.g., see Basso 1998; Fuster 1973). Of the 88 neurons tested, 33 had a delay signal (Fig. $2 B$ ). For 21 of the neurons the delay period firing rate was higher than baseline, and for the remaining 12 neurons it was lower than baseline [both elevated and suppressed delay signals are thought to play a role in cognitive operations (e.g., Funahashi et al. 1989; Fuster et al. 1982; Niki 1974)]. It has been proposed that delay signals related to a restricted range of target locations or saccade vectors may reflect spatially restricted attention, memory, or planning (e.g., Niki 1974; Niki and Watanabe 1976). Thus we tested most of the neurons that had delay signals with multiple target loca-

TABLE 1. Distribution of signal types

\begin{tabular}{|c|c|c|c|}
\hline $\begin{array}{c}\text { Signal } \\
\text { Type }\end{array}$ & $\begin{array}{l}\text { Number of } \\
\text { Neurons } \\
\text { Carrying } \\
\text { Signal }\end{array}$ & $\begin{array}{c}\text { Observed } \% \text { of } \\
\text { Neurons } \\
\text { Carrying } \\
\text { Signal* }\end{array}$ & $\begin{array}{c}\text { Corrected } \% \text { of } \\
\text { Neurons } \\
\text { Carrying } \\
\text { Signal } \dagger\end{array}$ \\
\hline Delay & 33 & 38 & 37 \\
\hline Periph. vis. & 40 & 45 & 50 \\
\hline Pre. burst & 41 & 47 & 34 \\
\hline Postsac. & 25 & 28 & 40 \\
\hline Pre. pause & 8 & 9 & 12 \\
\hline Gap incr. & 34 & 39 & 32 \\
\hline Gap decr. & 14 & 16 & 10 \\
\hline Fov. vis. & 7 & 8 & 14 \\
\hline Fix.-related & 20 & 23 & 24 \\
\hline
\end{tabular}

Periph., peripheral; vis., visual; pre., presaccadic; postsac., postsaccadic; incr., increase; decr., decrease; fov., foveal; fix., fixation. * Number of neurons carrying each signal type divided by 88 (the total number of neurons tested) and multiplied by 100 . $\dagger$ A function of conduction velocity (see text). 
TABLE 2. Distribution of neuron types

\begin{tabular}{lccc}
\hline \hline $\begin{array}{c}\text { Neuron } \\
\text { Type }\end{array}$ & $\begin{array}{c}\text { Number of } \\
\text { Neurons }\end{array}$ & $\begin{array}{c}\text { Observed \% of } \\
\text { Neurons* }\end{array}$ & $\begin{array}{c}\text { Corrected \% of } \\
\text { Neurons } \dagger\end{array}$ \\
\hline V. & 17 & 19 & 26 \\
M. & 18 & 20 & 9 \\
V.M. & 23 & 26 & 24 \\
Fov. V. & 4 & 5 & 4 \\
Fix. & 2 & 2 & 5 \\
\hline
\end{tabular}

V., Visual Neurons; M., Movement Neurons; V.M., Visuomovement Neurons; Fov. V., Pure Foveal Visual Neurons; Fix., Pure Fixation Neurons. * Number of neurons in each neuron type divided by 88 (the total number of neurons tested) and multiplied by 100 . $\dagger$ A function of conduction velocity (see text).

tions and found that $90 \%$ (26/29) did exhibit spatially restricted delay signals (spatial regions associated with the delay signals were contralateral for $80 \%$ of neurons, ipsilateral for $8 \%$, and on the vertical meridian for $12 \%$ ).

A neuron had a peripheral visual signal if its firing rate was elevated just after target onset (Vis epoch, Fig. 2A) compared with baseline in either visual or memory trials and/or if its firing rate during the delay period was greater in visual than in memory trials (indicative of a tonic visual response). A neuron had a presaccadic burst signal if, in either visual or memory trials, its firing rate just before saccade initiation (Pr epoch) exceeded both the baseline and the delay period firing rates. Forty neurons had a peripheral visual signal, and 41 had a presaccadic burst signal.

For comparison to previous studies (Bruce and Goldberg 1985; Segraves and Goldberg 1987), we sorted neurons into three types: Visual Neurons $(n=17)$ had a peripheral visual signal but not a presaccadic burst signal (Fig. 2C), Movement Neurons $(n=18)$ had a presaccadic burst signal but not a peripheral visual signal (Fig. 2D), and Visuomovement Neurons $(n=23)$ had both a peripheral visual signal and a presaccadic burst signal (Fig. 2E). Note that peripheral visual signals covered a wide range from primarily phasic (Fig. $2 C$, top) to primarily tonic (Fig. $2 C$, bottom). Neurons with peripheral visual signals or presaccadic burst signals often had delay signals as well; this occurred in $47 \%(8 / 17)$ of Visual Neurons, $44 \%(8 / 18)$ of Movement Neurons, and 57\% (13/23) of Visuomovement Neurons.

A neuron exhibited a postsaccadic signal if, in either visual or memory trials, its activity after a saccade (Po epoch, Fig. $2 A$ ) exceeded both the presaccadic activity and the baseline activity. Twenty-five neurons had a postsaccadic signal. For a minority $(32 \%, 8 / 25)$, this represented the continuation and acceleration of a presaccadic burst signal. The rest of these neurons exhibited a wide variety of phasic or tonic discharges after saccade termination. We did not test the neurons in total darkness and therefore do not know whether they were similar to the "Type I" postsaccadic neurons of Bizzi (1968). Neurons with postsaccadic signals are discussed further below, when we document the directional tuning of our neurons.

A neuron exhibited a presaccadic pause signal if it decreased its activity just before the saccade compared with during the delay and baseline periods. These were of interest because we thought that they might project preferentially to the rostral SC, which contains fixation neurons with similar presaccadic pauses. However, our FEF corticotectal neurons with presaccadic pauses $(n=8)$ as a class did not exhibit a rostral bias in their projection (other types of signals that did project in a biased way to the SC are described in detail below).

GAP-RELATED SIGNALS. We used the gap task to identify signals during gap periods that may be associated with cognitive processes such as fixation disengagement (e.g., Dias and Bruce 1994). A neuron had a gap increase signal if it increased its firing rate during the gap period (Gap epoch, Fig. 3A) compared with baseline (Base epoch). Of the 88 neurons tested, 34 exhibited this type of signal (Fig. 3B). We note three important characteristics of these neurons. First, many neurons with gap increase signals also exhibited peripheral visual signals $(62 \%$, $21 / 34)$, presaccadic burst signals $(65 \%, 22 / 34)$, or delay signals $(44 \%, 15 / 34)$ when tested with the delayed-saccade task. In terms of neuron types, gap increase signals were carried by $41 \%$ (7/17) of Visual Neurons, by $61 \%$ (14/23) of Visuomovement Neurons, and by 44\% (8/18) of Movement Neurons. Second, gap increase signals usually occurred even if target location was randomized. We tested 25 of the neurons with gap increase signals in a separate block of gap task trials in which the target appeared either within the visual or movement field of the neuron or, randomly on $50 \%$ of the trials, at the same eccentricity but $180^{\circ}$ opposite in direction. Most neurons $(88 \%, 22 / 25)$ still exhibited a gap increase signal in this block of trials. Third, when the target appeared outside the visual or movement field, elevated gap discharges quickly ceased after the target appeared (Fig. 3B). With respect to all of these discharge characteristics, our neurons with gap increase signals appear to be very similar to the FEF neurons described by Dias and Bruce (1994).

Another type of signal carried by FEF corticotectal neurons consisted of a decrease in activity during the gap period (Fig. $3 C$ ), i.e., a gap decrease signal. Neurons with this type of signal $(n=14)$ were not described previously (cf. Dias and Bruce 1994). They will be discussed in more detail below in relation to their pattern of projection onto the SC.

FOVEAL-RELATED SIGNALS. While testing neurons using the delayed-saccade and gap tasks, we noticed that 39\% (34/88) changed their firing rate at the start of fixation. This fovealrelated activity was quantified using the fixation task (Fig. 4A), and two kinds of signals were defined. A neuron had a fixationrelated signal (Fig. $4 B$ ) if its activity during the late blink period (Fix2 epoch, Fig. 4A) was different (greater or less than) baseline (Base epoch). This activity was not a foveal visual response for two reasons: first, it was not a foveal off-response because the change in activity persisted for hundreds of milliseconds after fixation spot disappearance; second, it was not a response to the diffuse light on the screen because activity during the Fix 2 epoch was different from baseline activity even though the fovea was illuminated with the same diffuse light during both periods. The only difference between the Base epoch and the Fix2 epoch was the requirement to maintain fixation at the center of the screen during the latter, and therefore we interpreted the type of activity shown in Fig. $4 B$ as related to the motor act of fixating. On the other hand, some neurons did carry signals clearly related to foveal visual stimulation. We considered a neuron to have a foveal visual signal (Fig. 4D) if it increased its activity just after fixation spot reappearance (VisOn epoch) compared both to baseline and to the late blink period. Twenty-four neurons had fixation-related or foveal visual signals (20 had fixation-related signals, 7 had 
foveal visual signals, and 3 had both). The remaining 10 neurons exhibited discharges during the fixation task that resisted simple classification.

We defined two mutually exclusive neuron types to separate our foveal-related neurons into visual and motor extremes. Two neurons were termed Pure Fixation Neurons (Fig. 4C) and were considered to fall at the motor end of the spectrum of signals, because their discharges were elevated relatively steadily above baseline throughout the entire trial period (i.e., during all 4 epochs Fix1, VisOff, Fix2, and VisOn of Fig. 4A). At the other extreme, four neurons appeared to be entirely visual, having a foveal visual signal but no fixation-related signal at all; these were called Pure Foveal Visual Neurons (Fig. 4D). Pure Foveal Visual Neurons simply discharged when the fovea was illuminated with a discrete spot of light and fell silent otherwise. Note that the lack of activity during the Fix 2 epoch (Fig. 4D) was not related to the act of steadily fixating because an identical lack of activity occurred during the Base epoch, when fixations and saccades were interspersed.

CONDUCTION VELOCITY, CELL SIZE, AND SAMPLING BIAS. Conduction velocity distributions for the 88 neurons tested on behavioral tasks and for all 138 neurons are shown in Fig. 5A (medians 30 and $26 \mathrm{~m} / \mathrm{s}$, respectively; not significantly different). We compared the conduction velocities of neurons carrying each type of signal or belonging to each defined neuron type to the conduction velocities of all the other neurons analyzed with behavioral tasks. The only significant results were that Movement Neurons had higher conduction velocities than other neurons (Fig. $5 B$; medians 43 vs. $25 \mathrm{~m} / \mathrm{s}, P=0.007$ ) and that the general class of all neurons carrying a presaccadic burst signal had higher conduction velocities than other neurons (Fig. $5 C$; medians $37 \mathrm{vs.} 21 \mathrm{~m} / \mathrm{s}, P=0.002$ ). These two categories of neurons, therefore probably had larger axons and cell bodies than the other neurons (Cullheim 1978; Gasser 1941; Kernell and Zwaagstra 1981).

When a neuronal population is made up of cells that vary in size, attempts to characterize the population with microelectrode recordings can be affected by sampling bias (Towe and Harding 1970). Larger neurons are more likely to be recorded from than smaller ones, because extracellular potentials of larger neurons are detectable further away from the cell body. We attempted to correct for this sampling bias using a previously established procedure (see METHODs). Figure $6 \mathrm{~A}$ shows the mean conduction velocities, in descending order, of neu-
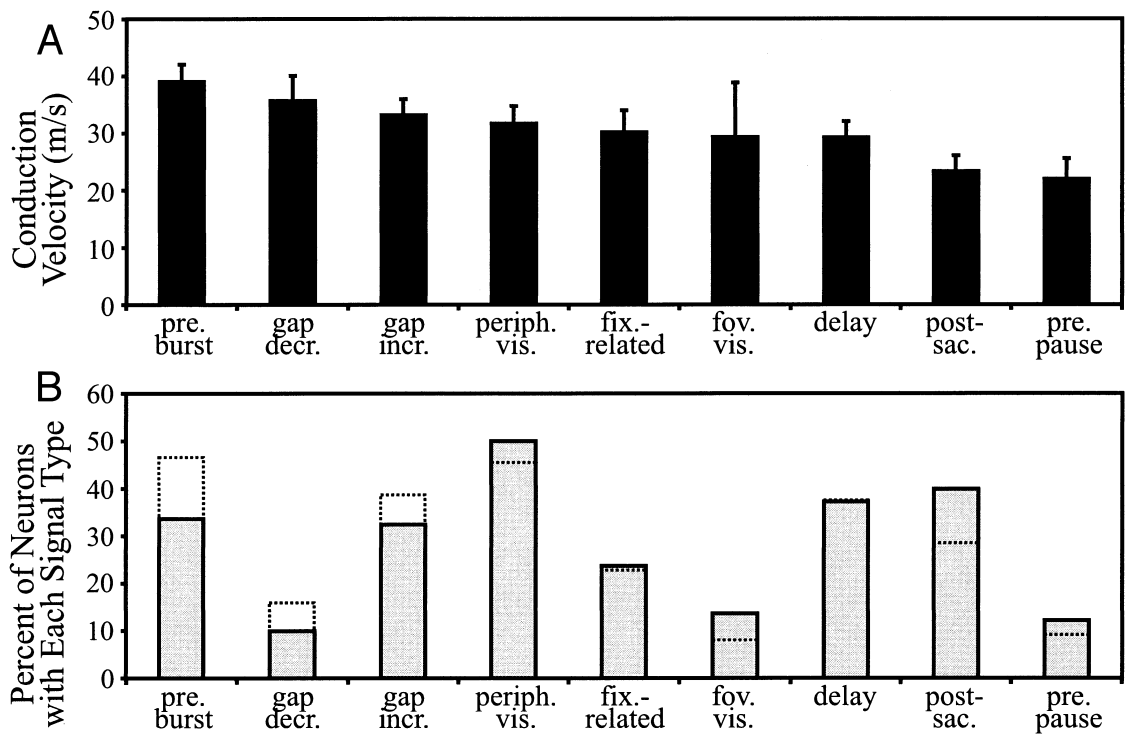

FIG. 6. Correcting for sampling bias using the method of Humphrey et al. (1978). A: distribution of conduction velocities (mean $\pm \mathrm{SE}$ ) for neurons carrying each type of signal, arrayed in decreasing order from left to right. B: observed and corrected percentages of neurons carrying each type of signal (legend at bottom right). $C$ and $D$ : distribution of conduction velocities $(C)$ and observed and corrected percentages $(D)$ of each defined neuron type. For key to abbreviations see legends of Tables 1 and 2.
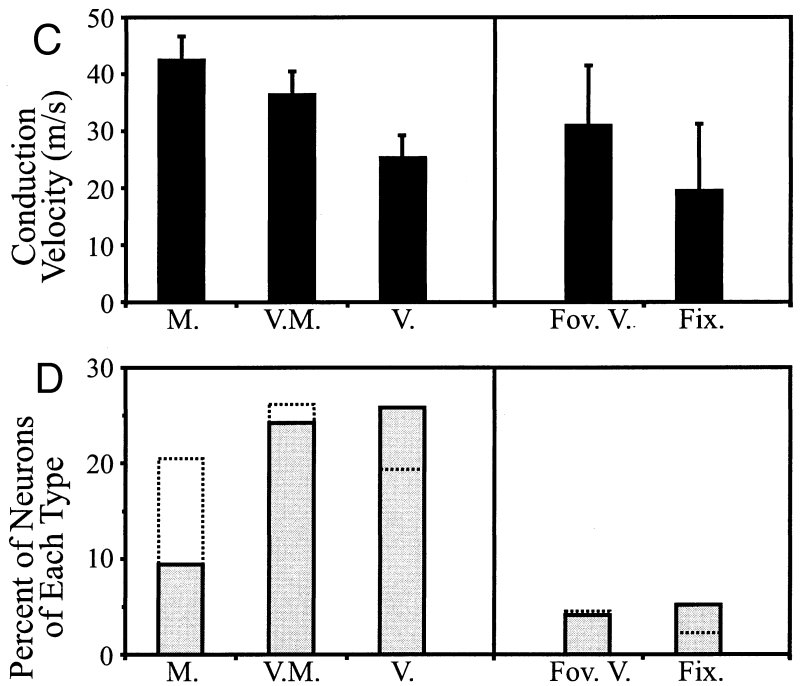
rons carrying each type of signal, and Fig. $6 B$ shows the observed (- - - -) and corrected percentages (畻) of these neurons. The percentages of neurons with relatively high conduction velocities tended to be decreased by the correction procedure, and the percentages of neurons with relatively low conduction velocities tended to be increased. To summarize all the corrected data, it appears that highly prevalent signals relayed from FEF to SC include peripheral visual signals (carried by $50 \%$ of neurons), postsaccadic signals (carried by $40 \%$ of neurons), delay signals (carried by $37 \%$ of neurons), presaccadic burst signals (carried by $34 \%$ of neurons), and gap increase signals (carried by $32 \%$ of neurons). The remaining signals appear to be carried by fewer than $25 \%$ of neurons projecting from $\mathrm{FEF}$ to $\mathrm{SC}$; in decreasing order of prevalence, these include fixation-related, foveal visual, presaccadic pause, and gap decrease signals (see Table 1 for exact percentages).

Figure $6, C$ and $D$, shows the results of performing the correction procedure on the neuron types defined above by the combinations of signals they carry. Mean conduction velocities of each neuron type are shown in Fig. $6 C$ (the 3 mutually exclusive neuron types related to peripheral visual stimulation and saccade generation are grouped together on the left, and the 2 mutually exclusive neuron types related to foveal visual stimulation and fixation are grouped together on the right). The observed percentages of Visual, Visuomovement, and Movement Neurons were similar (Fig. 6D, left, - - - -), but after correction for sampling bias (畻) it appears that both Visual and Visuomovement Neurons are about twice as prevalent as Movement Neurons in the population projecting from FEF to SC. After correction, Pure Foveal Visual Neurons and Pure Fixation Neurons (Fig. 6, $C$ and $D$, right) each appear to represent 4 and $5 \%$ of the population projecting from FEF to $\mathrm{SC}$, respectively. For exact percentages of each neuron type, see Table 2.

\section{Topographic organization of signals sent from FEF to SC}

DISTRIBUTIONS OF CELL BODIES IN FEF AND AXON TERMINALS IN SC. To analyze the distribution of corticotectal cell bodies, we first constructed a standard map of the FEF. Successful penetration entrance sites in the FEF (i.e., those yielding saccade-related corticotectal neurons) are shown for monkey $C$ in Fig. $7 A$ and for monkey $H$ in Fig. $7 D$. MRIs verified that penetration trajectories went through the rostral bank of the arcuate sulcus (MRI of monkey $C$ is shown in Fig. 7B). For monkey $H$ this was further confirmed by inspection of marking lesions and electrode tracks in histological sections (not shown). Low-threshold electrical stimulation $(<50 \mu \mathrm{A})$ within these recording sites or adjacent sites $(X$, Fig. $7, A$ and $D)$ evoked saccades at short latency (Fig. 7C). The amplitude of evoked saccades decreased from medial to lateral (Fig. 7C) and also from dorsal to ventral within a penetration (not shown). Our recording sites therefore were in the FEF as classically defined (e.g., Bruce et al. 1985; Robinson and Fuchs 1969). The recording sites tended to form a curve that paralleled the arcuate sulcus (Fig. 7, $A$ and $D$ ), undoubtedly because to yield corticotectal neurons the penetration trajectories had to intersect with or follow the contour of layer V (Fries 1984; Leichnetz et al. 1981), which runs parallel to the sulcus. For each monkey, we drew a curve representing the top edge of layer $\mathrm{V}$ onto the map of penetrations (Fig. 7, $A$ and $D$ ). This curve defined a mediolateral axis (Fig. $7 E$, top), with zero at the medial edge of the FEF (*, Figs. 7, $A$ and $D$ ) and with values increasing toward the more lateral FEF. The location of each recording site was described in relation to the mediolateral axis using orthogonal projection as shown schematically in Fig. 7D. Recording depth below the cortical surface defined a second, dorsoventral axis (Fig. 7E, top), with zero at the cortical surface and with values increasing down through the bank, parallel to the sulcus. Note that the mediolateral and dorsoventral axes used in this report are rotated from the conventional stereotaxic axes (Fig. 7E, bottom).

The estimated cell body locations for 131 of our corticotectal neurons are plotted on the standard map of the FEF in Fig. 8. The seven remaining neurons were not plotted because their depth measurements were uncertain. The FEF regions sampled in the two monkeys overlapped considerably and were of similar shape and areal extent (solid vs. dashed outlines in Fig. 8). The distribution of FEF corticotectal neurons from either monkey alone was patchy, due to clustering of corticotectal neurons and/or penetrations running only partway through layer V. Taken together, however, the data shown in Fig. 8 appeared to represent a reasonably homogeneous sampling across the FEF.

It should be noted that layer $\mathrm{V}$ in the FEF exhibits some curvature in the rostrocaudal dimension, too, and therefore a three-dimensional map would be required to represent the structure perfectly. This was beyond the scope of our study, however, and we feel that the two-dimensional approximation (Fig. 8) suffices for our purposes. Similarly, the SC is nearly always represented as a two-dimensional map (rostrocaudal and mediolateral axes) despite its curvature in a third dimension (dorsoventral axis) (e.g., Anderson et al. 1998; Ottes et al. 1986; Robinson 1972).

The rostral and caudal locations of SC stimulating electrodes for one experiment are shown with respect to the SC topographic map (Robinson 1972) in Fig. 9A. Marking lesions made through these electrodes (Fig. 9B) confirmed that their tips were located in the intermediate gray layer, and it appears that effective current spread was mostly confined to the SC (circles, Fig. 9, $A$ and $B$ ). We used the Electrode Preference Index (EPI, see METHODS) to estimate, for 108 neurons, whether projections were biased toward the rostral or the caudal SC. For the other 30 neurons, EPI was not calculated because either the rostral or the caudal electrode did not conduct currents reliably. The histograms of EPI for the two monkeys were similar (Fig. 9C); for each monkey, there were peaks at the extremes of the distribution, representing neurons activated only from the rostral (EPI $=-1)$ or the caudal $(\mathrm{EPI}=1) \mathrm{SC}$ electrode, and between the peaks there was a broad distribution of EPIs, representing neurons activated from both electrodes.

DISTRIBUTIONS OF TASK-RELATED SIGNALS IN CORTEX. Using the FEF standard map, we compared the FEF locations of neurons carrying each type of signal or belonging to each defined neuron type to the FEF locations of all the other neurons analyzed with behavioral tasks. Comparisons were made in the mediolateral and the dorsoventral directions (Student's $t$-test or Mann-Whitney rank sum tests were used as appropriate, and because we tested the data twice, along orthogonal axes, the significance criterion was adjusted to $P<$ $0.05 / 2=0.025)$. Signals putatively related to cognitive oper- 


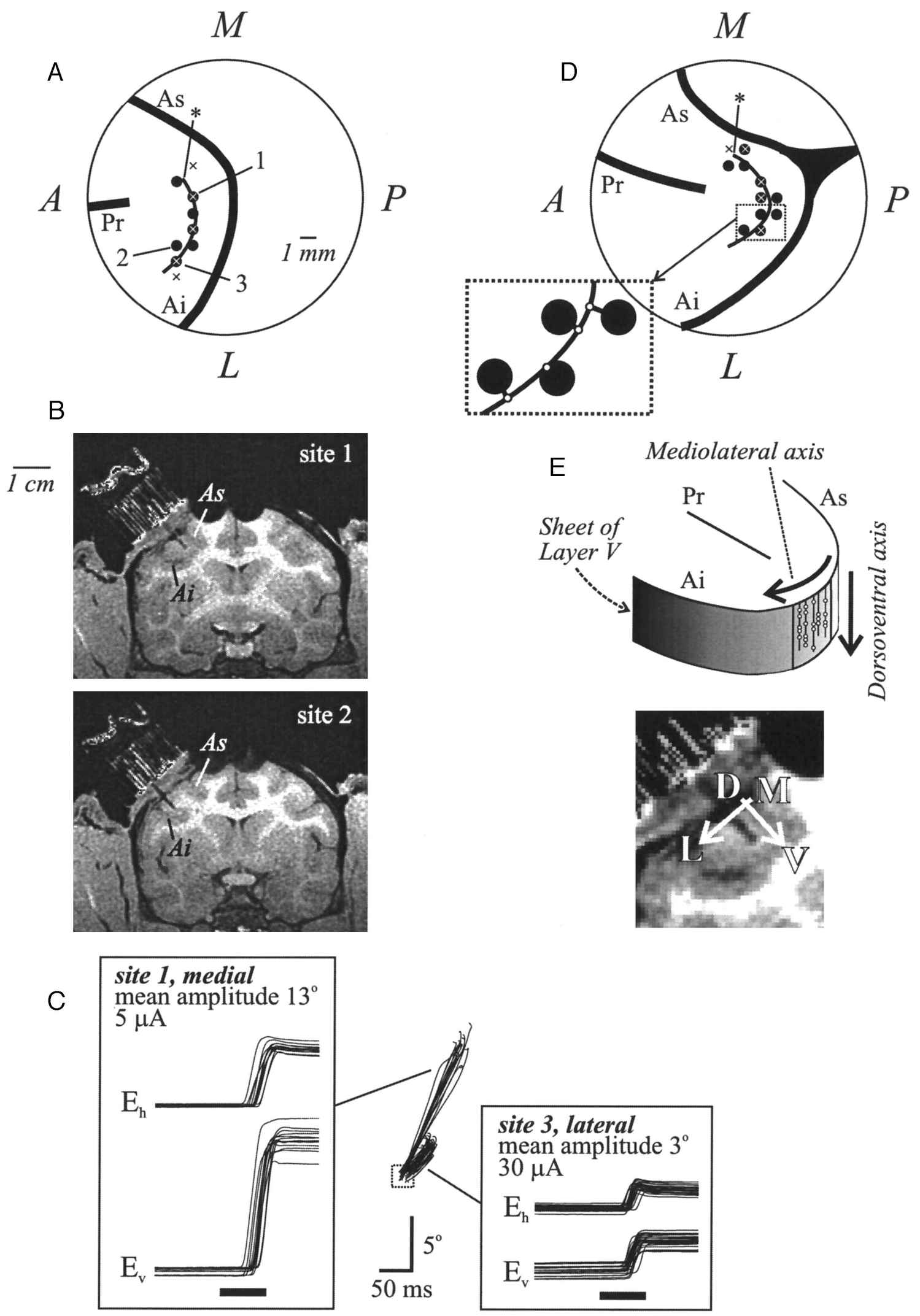




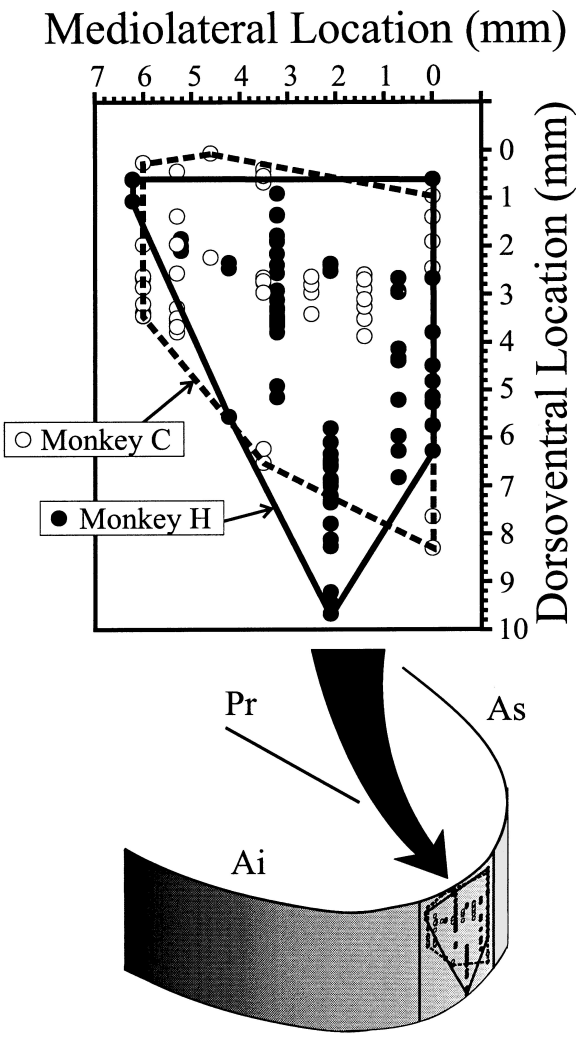

FIG. 8. Distribution of cell body locations (circles) in the FEF for each monkey. Note that 131 neurons were plotted, but due to clustering of the recorded neurons many of the symbols are occluded by others. Polygons depict the general area of sampling for each monkey. Explicit diagram of how this graph corresponds to gross anatomy is shown at bottom.

ations (delay and gap increase signals) were carried by neurons located more dorsally in the FEF than other neurons (Fig. 10A; medians 2.7 vs. $3.6 \mathrm{~mm}$ along the dorsoventral axis, $P<$ 0.001). This dorsal bias was significant for each of the component signal types, too (i.e., for the neurons carrying a delay signal as well as for those carrying a gap increase signal). Neurons carrying a peripheral visual signal but not a presaccadic burst signal (Visual Neurons) were biased laterally in the FEF compared with other neurons (Fig. 10B; medians 3.5 vs.
$2.5 \mathrm{~mm}$ along the mediolateral axis, $P=0.018$ ). In contrast, neurons carrying a presaccadic burst signal but not a peripheral visual signal (Movement Neurons) were biased medially in the FEF compared with other neurons (Fig. 10C; medians 2.1 vs. $3.2 \mathrm{~mm}$ along the mediolateral axis, $P=0.010$ ). No other cortical topographies of signals or defined neuron types were found.

DISTRIBUTIONS OF TASK-RELATED SIGNALS PROJECTING TO SC. Using the EPI measure, we compared the axon termination locations of neurons carrying each type of signal or belonging to each defined neuron type to the axon termination locations of all the other neurons analyzed with behavioral tasks (Student's $t$-test or Mann-Whitney rank sum tests were used as appropriate; $P<0.05$ criterion). Three cases of projection bias were found. First, Movement Neurons projected more caudally in the SC than did other neurons (Fig. 10D; median EPIs 0.44 vs. -0.09 respectively, $P=0.043)$. Second, neurons carrying a gap decrease signal projected more rostrally than did other neurons (Fig. $10 E$; median EPIs -1.0 vs. $0.114, P=0.017$ ). Third, Pure Foveal Visual Neurons projected more rostrally than did other neurons (median EPIs -1.0 vs. 0.084, $P=$ 0.034). This bias of Pure Foveal Visual Neurons should be interpreted cautiously, however, due to small sample size. In light of a prediction by Dias and Bruce (1994) that FEF neurons with elevated gap activity may project uniformly over the SC, we note that our data are consistent with this prediction in that the EPI distribution of neurons with gap increase signals was broad and not biased rostrally or caudally (not shown).

FEF neurons that projected in a biased manner to rostral SC (Fig. 10, $E$ and $F$ ) were similar in many ways to neurons within the rostral SC. First, both rostral SC neurons and our FEF corticotectal neurons with a gap decrease signal are known to lower their activity during gap periods (cf. Dorris and Munoz 1995). Rostral SC neurons often have foveal visual receptive fields, often cease their activity when a saccade begins, and often have elevated activity after a saccade ends (Munoz and Wurtz 1993a), and our FEF corticotectal neurons that carried a gap decrease signal were comparable, as follows. When neurons with a gap decrease signal were tested using the fixation task, we found that $43 \%(6 / 14)$ had a foveal visual receptive

FIG. 7. Recording sites in the FEF. A: recording chamber for monkey $C$. Entrance sites of penetrations that yielded saccaderelated corticotectal neurons are shown with dots. Curved line between the penetration sites is the estimated top edge of layer V. Asterisk shows the medial edge of the functionally defined FEF. Sites where reference electrodes were inserted for MRI are labeled 1 and 2 , respectively. $\times$, sites where saccades were electrically evoked at low threshold $(<50 \mu \mathrm{A}$; these include sites labeled 1 and 3 , discussed further in $C$ ). $B$ : examples of coronal plane MRIs for monkey $C$, showing the recording chamber and grid (top left in each MRI) and reference electrodes (dark lines extending from chamber into the brain). Grid holes were visualized by filling the grid and chamber with betadine ointment $\sim 1 \mathrm{~h}$ before taking the MRI. Top: reference electrode at site 1 as labeled in A. Bottom: reference electrode at site 2. $C$ : examples of electrically evoked saccades. In the center, trajectories of saccades evoked from 2 recording sites (labeled 1 and 3 in $A$ ) are illustrated. Dotted box shows the fixation window (monkey was trained to keep its eyes still within this window, but stimulation moved the eyes out of it). In the large boxes at left and right, saccades from each site are decomposed into horizontal $\left(E_{\mathrm{h}}\right)$ and vertical $\left(E_{\mathrm{v}}\right)$ components and plotted against time; bars below the eye traces depict stimulation onset and duration. The mean amplitudes of saccades evoked from each site, and the suprathreshold currents used for evoking these saccades, are noted. Current threshold was $3 \mu \mathrm{A}$ at site 1 and $20 \mu \mathrm{A}$ at site 3. Amplitude and time scales at bottom. D: recording chamber for monkey $H$. The method of projecting penetration entrance sites orthogonally onto the curve of layer $\mathrm{V}$ is diagrammed in the dotted-outline box (projected locations are shown as white dots). E: standard map of the FEF. At top, layer V is depicted as a sheet, electrode trajectories are depicted as vertical lines running tangential to this sheet, and recorded cells are depicted as small circles (note, these are not actual data, but are shown only to help illustrate the method). Each cell was mapped in 2 dimensions by describing its position with respect to the mediodorsal axis, which is the top edge of layer $\mathrm{V}$ as drawn in $A$ and $D$, and the dorsoventral axis, which is parallel to the penetration trajectory. Zero on the mediolateral axis is the medial edge of the FEF ( $*$ in $A$ and $D$ ), and zero on the dorsoventral axis is the top of the cortex. At bottom, the mediolateral and dorsoventral axes are shown in reference to a coronal section of the FEF, which was cropped from the top MRI in $B$ and enlarged. Ai, As, inferior and superior limbs of the arcuate sulcus, respectively; Pr, principal sulcus; A, anterior; P, posterior; M, medial; L, lateral; D, dorsal; $\mathrm{V}$, ventral. 
field, exhibiting an excitatory foveal on-response, an inhibitory foveal off-response, or both. In the delayed-saccade task, $29 \%$ (4/14) of neurons with a gap decrease signal ceased firing just before saccade initiation (i.e., they exhibited a presaccadic pause signal), and $43 \%(6 / 14)$ had elevated firing rates after saccade termination (i.e., they exhibited a postsaccadic signal). Finally, although the rostral bias of Pure Foveal Visual Neurons is arguable due to small sample size, it is notable that their defining signal, i.e., a foveal visual response, is a type of signal also present in many rostral SC neurons (Munoz and Wurtz 1993a).

VISUAL AND MOVEMENT FIELD ECCENTRICITY. Most of the neurons evaluated using behavioral tasks $(90 \%, 79 / 88)$ had spatially restricted visual receptive fields, movement fields, or
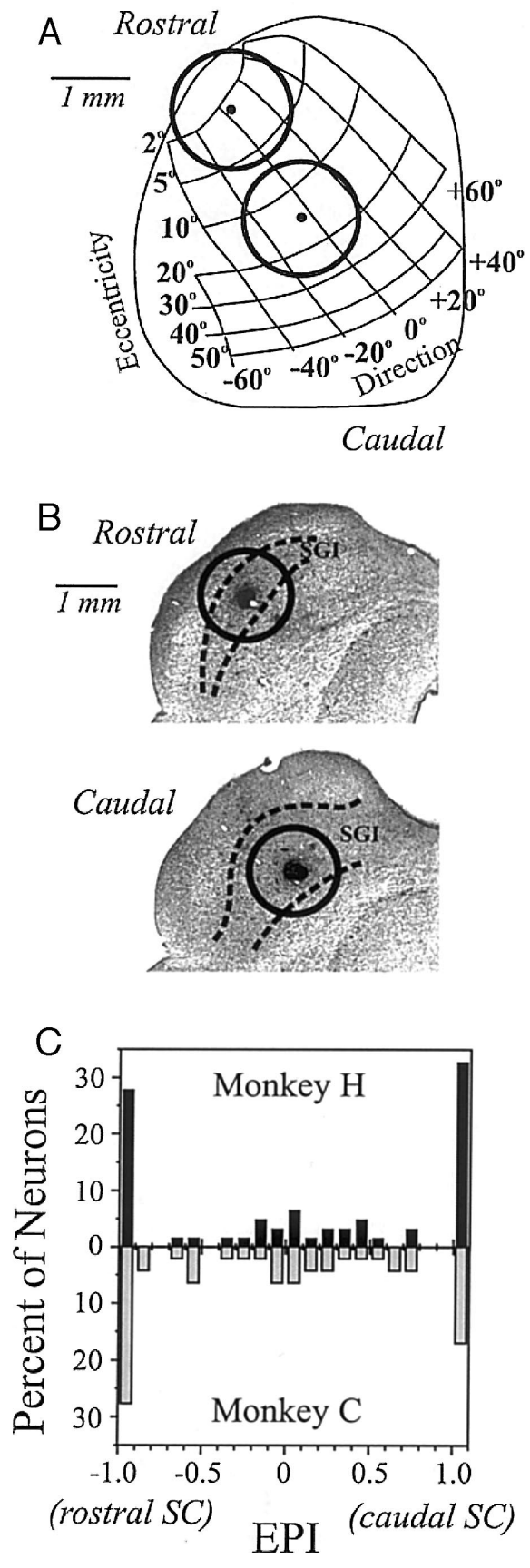

both. The best location of each field (see METHODS) was described in polar coordinates, yielding a best eccentricity and best direction. There were too few data to analyze topographies of visual and movement fields separately, so we combined the data (this appeared reasonable, because for neurons with both a peripheral visual signal and a presaccadic burst signal, their visual and movement fields overlapped considerably such that there was nearly always a single best location for both types of activity). Occasionally we could determine only a best eccentricity or a best direction, but not both.

Seventy-two neurons exhibited a distinct best eccentricity of their visual or movement field. To search for topographic organization, we examined whether a neuron's best eccentricity varied as a function of its mediolateral and dorsoventral location in FEF using multiple linear regression, and this was highly significant $(R=0.64, P<0.001)$. We also tried using the logarithm (base 10) of eccentricity as the dependent variable, and this worked equally well $(R=0.64, P<0.001)$. Therefore the choice of dependent variable, eccentricity or $\log _{10}$ (eccentricity), was arbitrary. We decided to use the latter because eccentricity seems to be represented in the brain most often logarithmically [for example in SC (Ottes et al. 1986) and primary visual cortex (Schwartz 1994)]. The planar fit (Fig. $11 A$ ) was described by $\log _{10}$ (eccentricity) $=1.748-0.160$ (mediolateral location) - 0.0546 (dorsoventral location). $\log _{10}$ (eccentricity) decreased significantly both from medial to lateral $(P<0.001)$ and from dorsal to ventral $(P=0.036)$ in the FEF ( $P$ values are for the 2 coefficients in the planar equation). Isoeccentricity contours derived from this planar regression are shown in Fig. 11B, providing for the first time an explicit two-dimensional map of visual and movement field eccentricity in the FEF.

Next we wanted to determine how this FEF eccentricity map projected onto the known eccentricity map running along the rostrocaudal axis of the SC. As shown with the scatter plot in Fig. $11 C$, there was a general trend for neurons in medial and dorsal FEF (at top right in the scatter plot) to project to caudal SC $(\bigcirc)$ and for neurons in lateral and ventral FEF (bottom left in scatter plot) to project to rostral SC (-). To quantify this, we first attempted to use multiple linear regression, but the data were not parametric (normality and constant variance tests

FIG. 9. Stimulation sites in the SC. A: examples of rostral and caudal stimulating electrode locations (dots) in the $\mathrm{SC}$ with respect to its topographic map (Robinson 1972). Rostral and caudal directions, as well as isoeccentricity and isodirection contours, are labeled. $B$ : depths of the electrode tips for the SC penetration sites of $A$, shown in coronal sections. Marking lesions (black marks at the middle of circles) were made at the end of the experiment through the rostral (top) and caudal (bottom) electrodes. The approximate dorsal and ventral boundaries of the stratum griseum intermediale (SGI), as determined by examination of successive cell- and myelin-stained sections through the entire rostrocaudal extent of the SC, are shown with curved dotted lines. In the caudal section the marking lesion appears relatively deep and the SGI relatively wide; these are artifacts due to the plane of section being somewhat oblique to the SC surface. In $A$ and $B$, estimates of the mean effective current spread are shown with circles, as calculated using the equation Distance $=(\text { Current } / K)^{0.5}(\mathrm{Te}$ hovnik 1996), where $K \cong 381 \mu \mathrm{A} / \mathrm{mm}^{2}$ (Sommer and Wurtz 1998a). Mean current thresholds for antidromic activation were $208 \pm 144 \mu \mathrm{A}$ from the rostral electrode and $198 \pm 122 \mu \mathrm{A}$ from the caudal electrode, suggesting that we activated neural elements on average within $0.74 \pm 0.62 \mathrm{~mm}$ from the rostral electrode tip and within $0.72 \pm 0.57 \mathrm{~mm}$ from the caudal electrode tip. $C$ : distribution of the rostrocaudal extent of axon termination in the SC (quantified with Electrode Preference Index, EPI) for each monkey. EPI histogram for monkey $C$ (四) is inverted and placed directly below that from monkey $H(\square)$ to permit direct comparison. 


\section{Topographies of Cell Bodies}

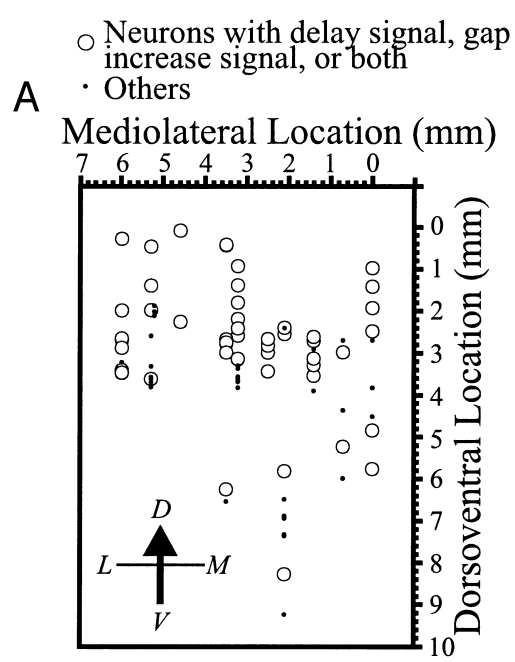

$\circ$ Visual Neurons

B - Others

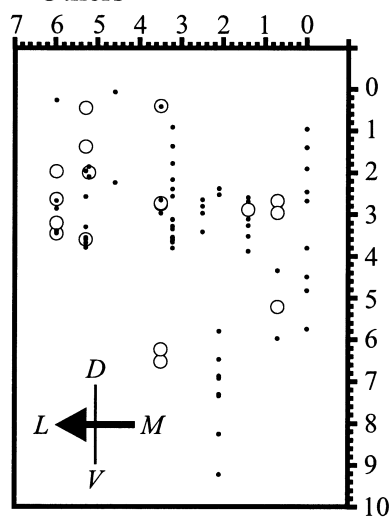

O Movement Neurons

C

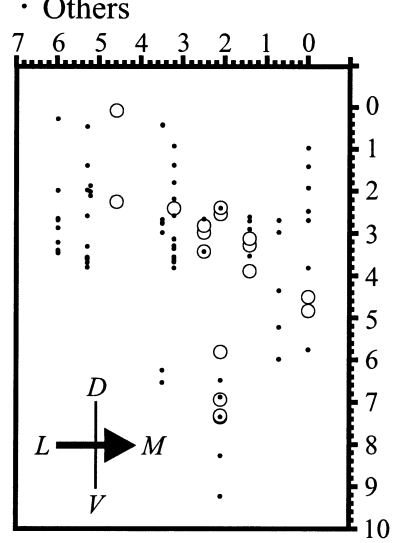

\section{Topographies of Axon Terminations}
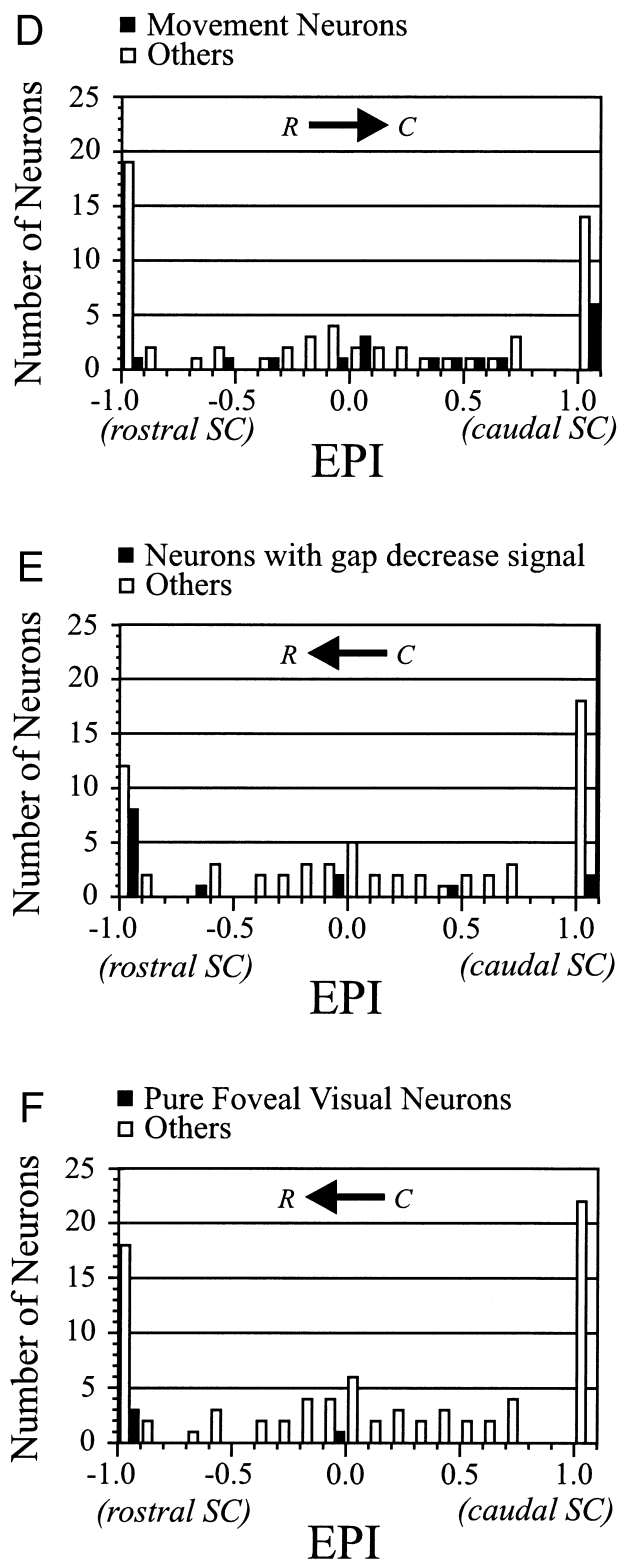

FIG. 10. Topographies of signals and neuron types with respect to cell body location in FEF (left column) and axon termination region in SC (right column). Arrow in each panel summarizes the direction of significant topographic bias: D, dorsal; V, ventral; M, medial; L, lateral; R, rostral; C, caudal. Plotting conventions for $A-C$ are the same as in Fig. 8, but only the 88 neurons fully tested on behavioral tasks are included here. $A$ : neurons with a putative cognitive function (those carrying a delay signal, a gap increase signal, or both) were biased dorsally in the FEF compared with other neurons. Visual Neurons were biased laterally $(B)$ and Movement Neurons were biased medially $(C)$ in the FEF compared with other neurons. The projections of Movement Neurons were biased caudally $(D)$ in the SC compared with the projections of other neurons. In contrast, the projections of neurons carrying a gap decrease signal $(E)$ and Pure Foveal Visual Neurons $(F)$ were biased rostrally in the SC compared with the projections of other neurons. failed). Therefore we used multiple nonparametric correlation analyses. Seventy-two test axes were defined, oriented in $5^{\circ}$ increments around $360^{\circ}$, where the $0^{\circ}$ test axis pointed rightward along the mediolateral axis, the $90^{\circ}$ test axis pointed upward along the dorsoventral axis, and so forth. For each test axis, the locations of neurons in the FEF were projected orthogonally onto the axis. Then the locations of the neurons on this axis were compared with the EPIs of the neurons using Spearman correlation (significance levels were Bonferroni ad- justed for the multiple comparisons). Correlation was highest along the test axis angled $30^{\circ}$ up from the mediolateral axis $(R=0.47, P<0.001$; see thick, solid arrow in Fig. 11C). Notably, this was closely aligned to the direction of steepest change in eccentricity in the FEF (as quantified by the direction of the gradient of the plane in Fig. $11 A$, which was $19^{\circ}$ up from the mediolateral axis; drawn with thin, dashed arrow in Fig. $11 C$ ). This alignment suggested that FEF neurons representing more eccentric space tend to project more caudally in the SC, 
A

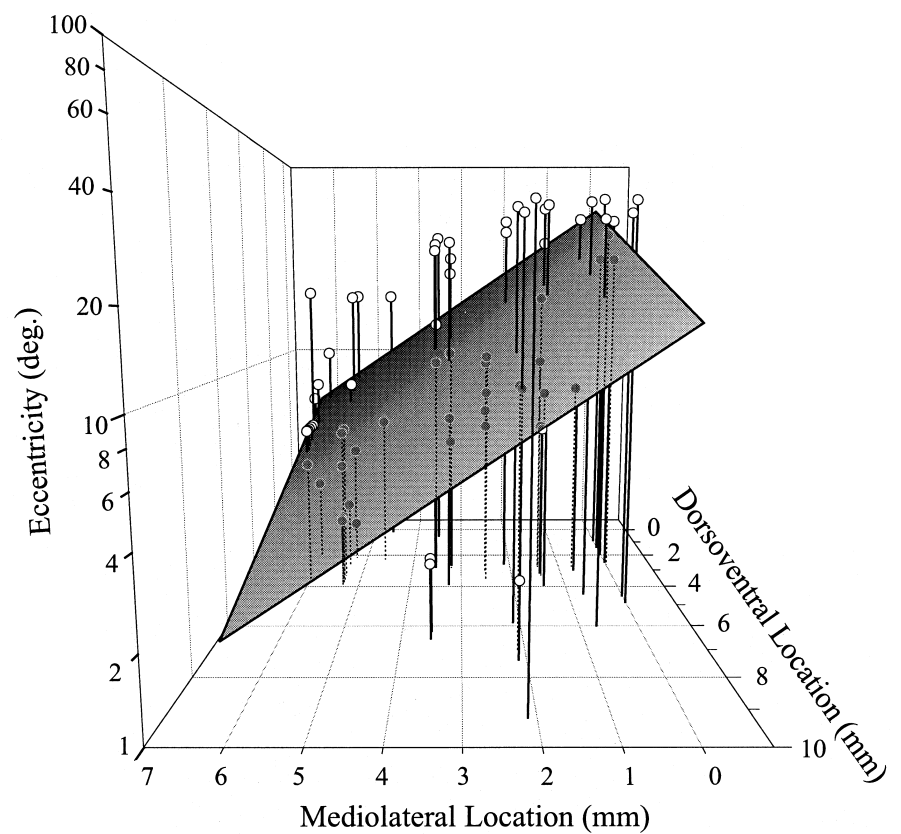

B Eccentricity Map

Mediolateral Location ( $\mathrm{mm}$ )

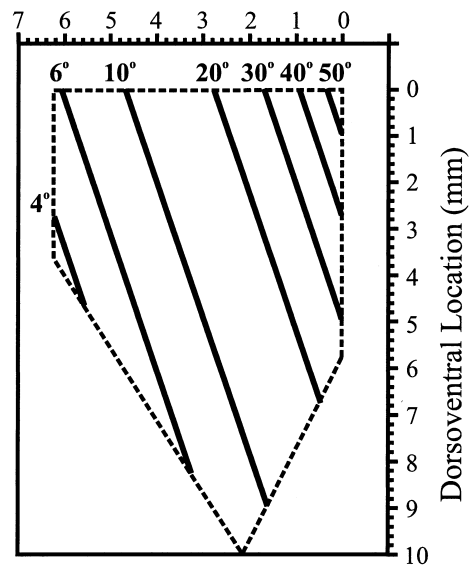

C Projection Map Mediolateral Location $(\mathrm{mm})$

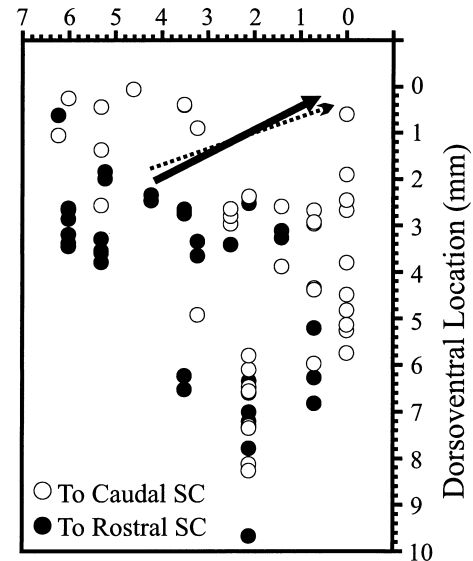

FIG. 11. Topographies of visual and movement field eccentricity and of projection onto the SC. A: the visual or movement field eccentricity for each neuron is plotted ( $z$-axis, $\log$ scale) as a function of the neuron's location within FEF, and a plane is fit to the data. Data that would be occluded by the plane are depicted with gray circles and dotted drop-lines. View is from the fundus looking up toward the lip of the arcuate sulcus; lateral is to the left and medial is to the right. Note that eccentricity decreases both from medial to lateral and from dorsal to ventral within the FEF. $B$ : eccentricity map of corticotectal neurons in the FEF is shown. Isoeccentricity contours are derived from the equation for the planar regression. Map is confined to the region within the dotted boundary, which represents the range of neuron locations that contributed to the map. $C$ : projection map of corticotectal neurons in FEF. Scatter plot shows the locations of FEF corticotectal neurons that projected caudally $(\mathrm{O}, n=41$; EPI $>0.33)$ or rostrally in the SC $(\bullet, n=39$; EPI $<-0.33)$. Other neurons $(n=26$; $-0.33<\mathrm{EPI}<0.33)$ were omitted from the illustration for clarity. The thick, solid arrow shows the direction of optimal correlation between cell body location and projection to the SC (angled $30^{\circ}$ up relative to mediolateral axis). The thinner, dashed arrow shows the direction of the eccentricity gradient (angled $19^{\circ}$ up relative to the mediolateral axis). and this relationship was confirmed by correlation analysis: the best eccentricity of a neuron's field was directly correlated with its projection zone across the SC as measured by EPI (Pearson $R=0.40, P<0.001)$, and $\log _{10}$ (eccentricity) versus EPI provided an even better correlation [Pearson $R=0.46$; equation of linear regression was $\log _{10}$ (eccentricity) $=1.06+$ $0.29 \mathrm{EPI}]$. Using this linear regression we constructed a diagram in Fig. 12 to explicitly illustrate how the eccentricity signals carried by FEF afferents appear to terminate across the eccentricity map of the SC. Both the eccentricity map of FEF terminations and the SC map appear to be logarithmic and are similarly scaled. The FEF afferent map, however, appears to be shifted $\sim 1 \mathrm{~mm}$ rostral to the SC map; this shift may be an artifact of occasionally activating axon main fibers rather than axon terminals (see DISCUSSION).

VISUAL AND MOVEMENT FIELD DIRECTION. Sixty-seven neurons exhibited a distinct best direction of their visual or movement fields. To summarize the general distribution of best directions, they were oriented contralaterally for $69 \%$ of neurons (46/67), ipsilaterally for $9 \%(6 / 67)$, and vertically for $22 \%(15 / 67)$. Too few corticotectal neurons were encountered in each penetration to discern whether there was a significant rotation of best direction with depth of penetration, as was described for stimulation-evoked saccades by Bruce et al. (1985). In some penetration sites we could reliably obtain nearly the same best direction from day to day within a narrow range of depths, which suggests that an underlying map of direction might exist. However, we could not detect any systematic topographic layout of best direction in our data.

Many ipsilaterally tuned neurons in the FEF carry postsaccadic signals that have been hypothesized to be used in updating a local retinotopic frame of reference (Goldberg and Bruce 1990). Perhaps consistent with this hypothesis, we note that only one of the ipsilaterally tuned neurons in our sample of efferent neurons exhibited a postsaccadic signal. Other types of signals found in our ipsilaterally tuned neurons included peripheral visual signals ( 3 neurons) and presaccadic burst signals (3 neurons).

OMNISPATIAL VISUAL AND MOVEMENT FIELDS. Ten percent of the neurons (9/88) had unrestricted visual and movement 


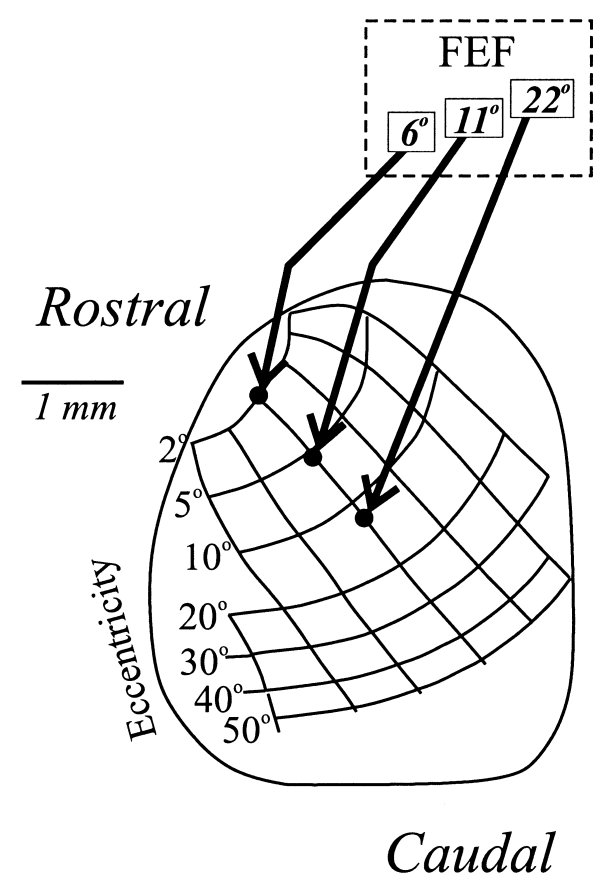

FIG. 12. Projection of FEF neurons representing various eccentricities onto the SC eccentricity map. An explicit diagram of the projection from FEF to SC was constructed using the linear regression equation $\log _{10}$ (eccentricity) $=$ $1.06+0.29 \mathrm{EPI}$. The 3 dots on the SC map represent, from rostral to caudal, EPI $=-1.0,0.0$, and 1.0. The EPI $=-1.0$ point, representing the rostral stimulating electrode location, which always was within $3^{\circ}$ eccentricity on the $\mathrm{SC}$ map, was arbitrarily placed on the $2^{\circ}$ isoeccentricity line; the EPI $=1.0$ point, representing the caudal stimulating electrode location, was placed at $\sim 12^{\circ}$ eccentricity, which was the mean location for caudal electrodes (see METHODS); the EPI $=0.0$ point was placed midway between the other 2 points.

fields; i.e., they exhibited approximately equal visual- or movement-related activity over all of the testing space. We found no topographies of cell distribution in the FEF or of projection across the $\mathrm{SC}$ for these neurons. The most interesting property of these neurons was that nearly all (8/9) exhibited postsaccadic signals and three also carried fixation-related signals. Less common signal types in these neurons included peripheral visual signals (1 neuron) and presaccadic burst signals (1 neuron). A speculative role for these omnispatial neurons therefore would be to help terminate all saccades by projecting onto inhibitory interneurons in the SC that act to suppress the firing of movement-related neurons.

\section{I S C U S S I O N}

\section{Signal flow from FEF to SC}

The first goal of this study was to evaluate the composition of signals coursing from FEF to the SC. We found that the FEF engages in an exuberant narration during oculomotor behavior, telling the SC about nearly everything that occurs. Signals relating to fixation, to visual stimulation, to possible cognitive operations, and to saccade initiation and termination are all relayed from FEF to SC. Notably, all these types of signals are carried by the general population of FEF neurons, as well (e.g., Bruce and Goldberg 1985; Funahashi et al. 1989; Hanes et al. 1998; Schall 1991). We see no compelling evidence that any signal type is over- or underrepresented at the output of FEF relative to the general population of signals in FEF.
In contrast, Segraves and Goldberg (1987) concluded that purely saccade-related signals are overrepresented and purely visual-related signals are underrepresented in the output of FEF relative to the general population of signals within FEF. There are several possible reasons why our results differ from those of Segraves and Goldberg (1987) in this respect. The most important reasons may be related to topography: we found that purely saccade-related output neurons (Movement Neurons) tend to be concentrated in medial FEF (Fig. 10C) and tend to project preferentially to caudal SC (Figure 10D). If Segraves and Goldberg (1987) focused their recordings in medial FEF or their stimulations in caudal SC, this would have led to a larger proportion of purely saccade-related neurons in their sample than in ours. Although we suspect that this occurred, Segraves and Goldberg (1987) did not publish plots of their recording or stimulation sites. Assuming they did record primarily in medial FEF, we would agree with them that output is largely saccaderelated for this particular part of FEF. Even within medial FEF, however, it does not necessarily follow that there is an enrichment of saccade-related signals at the output compared with the general population. It could be that in medial FEF a large percentage of neurons in all laminae have saccade-related discharges. Regarding the lack of purely visual-related neurons in the sample of Segraves and Goldberg (1987), this too might be explained if they focused their recordings in medial FEF rather than in the visually rich lateral region (cf. Fig. 10B).

We found that saccade-related neurons are the largest corticotectal neurons of the FEF (Fig. 5, $B$ and $C$ ) and thus are likely to be oversampled relative to other corticotectal neurons. This probably did not cause the differences between our study and that of Segraves and Goldberg (1987), however. Oversampling of larger neurons should have affected both studies equally, and yet even in our uncorrected data there were fewer neurons primarily related to saccades $(20 \%, 18 / 88)$ than in their data $(53 \%, 27 / 51)$. Therefore we suspect that topographic factors, as described above, were the main reasons for differences between the studies.

It should be noted that there were subtle differences in the way that we and Segraves and Goldberg (1987) classified neurons. The two studies used different methods of detecting signals (we used statistical analyses and they used visual inspection) and some different tasks (e.g., we used randomly interleaved visual and memory forms of the delayed-saccade task and they did not, and they used a task in which a peripheral visual stimulus was presented that was not the target of a saccade and we did not). Nevertheless, in general it seemed to us that our Visual, Visuomovement, and Movement Neuron categories were similar to their categories of the same names. Also, our definitions of neurons with postsaccadic signals and foveal-related signals were about the same as theirs. They did not classify neurons in terms of their delay signals, and they did not test their neurons for gap signals, so comparisons between the studies with respect to these categorizations were not possible.

The only other study of FEF corticotectal signals was performed in cat by Weyand and Gafka (1998a,b), who directly compared these FEF output signals to the general population of FEF signals recorded in the same animals. We agree with their general finding, that FEF corticotectal signals are very similar to signals in the general FEF population.

Neuronal recordings in which projections are identified are 
crucial for testing models of how brain structures interact. In particular they help to evaluate cognitive models (Fuster 1997), which posit that signals are processed in stages (e.g., Miller 1988; Taylor 1976). What do our data suggest about modeling the relationship between FEF and SC? A major type of processing in FEF seems to be the transformation of visual signals into movement commands (Bruce and Goldberg 1985). There are two ways to represent this in terms of cognitive models. In a discrete multistage representation, only the output of the transformation, a movement command, is passed on to the next stage (Fig. 13A). In a continuous multistage representation, signals at any point in the transformation can go to the next stage (Fig. 13B). We found that purely visual-related and purely saccade-related signals, as well as signals representing possible intermediates from vision to movement, are all found in the output of FEF. Therefore we think that the FEF and SC are best modeled as forming a continuous multistage system (Fig. 13B): visual signals may be transformed into movement commands within the FEF, but they also are relayed straight to the SC, where they again become susceptible to visuomotor transformation.

\section{Comparison to other cortical output systems}

Is broad diversity of output signals, and similarity between the output signals and the general population of signals within a brain region, unique to the FEF? No, and in fact it may be a common principle in brain organization. Signals carried by neurons in primary motor cortex that are known to project into the spinal cord (pyramidal tract neurons) are only mildly different from signals carried by the general population of motor cortex neurons (reviewed by Porter and Lemon 1993); for example, $40-50 \%$ of general motor cortex neurons are modulated by static force as compared with $62 \%$ of pyramidal tract neurons. Both populations are extremely diverse in signal content (Porter and Lemon 1993). Also, a great diversity of signals is sent from the lateral intraparietal area to the SC (Paré and Wurtz 1997), including peripheral visual signals, delay signals, and presaccadic burst signals, and these signals are similar to those found generally in the lateral intraparietal area (cf. the review of Andersen and Gnadt 1989).

\section{A Discrete Multistage}

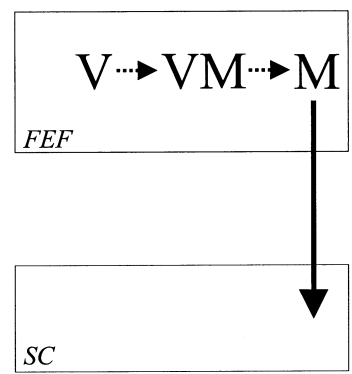

\section{B Continuous Multistage}

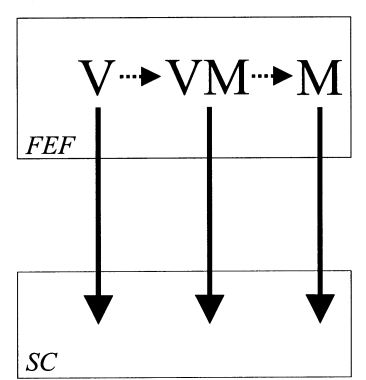

FIG. 13. Schematics illustrating 2 models of signal flow from FEF to SC: the discrete multistage model $(A)$ and the continuous multistage model $(B)$. Solid lines with arrows indicate signal transmission from FEF to SC. Dashed lines with arrows indicate hypothesized signal transformations within the FEF. $\mathrm{V}$, peripheral visual signal; VM, visuomotor signal (perhaps represented in the activity of Visuomovement Neurons or in the general class of neurons carrying delay signals) (see Bruce and Goldberg 1985); M, movement signal (i.e., presaccadic burst signal).
Some exceptions to this principle have been reported, as well. However, any conclusion that output signals represent only a subset of signals in the source area depends on a negative result (namely, that certain signals present in the general population are not part of the cortical output). This may be interpretable if a large sample of antidromically activated neurons is quantitatively analyzed, but that has not been the case for recent claims of signal specificity in cortical outputs [e.g., visual and polysensory cortex to SC, $n=8-22$ neurons analyzed per area in Wallace et al. (1993); MT to pretectum, $n=41$ neurons in Ilg and Hoffman (1993); V1 to MT, $n=9$ neurons in Movshon and Newsome (1996)]. Furthermore, it would be important to provide evidence that the entire cortical region of interest was sampled because high specificity in output signals might be only a local property, caused by topographic organization of a region's output signals as was found in the present study.

\section{Correction for sampling bias}

To estimate the composition of signals flowing from FEF to SC as carefully as possible, we tried to account for sampling bias caused by cell size variation. Did this procedure, originally developed for motor cortex data (Humphrey and Corrie 1978), succeed when adapted to our data? We tested this by seeing whether we could better predict the actual distribution of corticotectal neuron sizes in the FEF using the corrected, rather than the experimentally observed, conduction velocity data. We transformed the corrected and observed distributions of conduction velocities into estimated cell diameter distributions and then compared each to the known distribution of FEF corticotectal cell diameters (Fries 1984). Using the relationship Diameter $=a$ (Conduction Velocity $)^{b}$, we set $b$ to a physiologically relevant value (from 0.4 to 0.9 in increments of 0.1 ) (Kernell and Zwaagstra 1981) and then varied the coefficient $a$ until optimal fit was found between the estimated and actual cell diameter distributions (judged by least sum of squared errors). The overall best fits are shown in Fig. 14A, and the least sum of squared errors for each value of $b$ is shown in Fig. $14 B$. The cell diameter distributions derived from the corrected data always provided the better fit to the actual distribution. We conclude therefore that application of the correction procedure did counter some of the sampling bias and thus led to a more realistic estimate of the proportions of each signal type that leave the FEF.

\section{Delay, gap, and fixation signals}

We found that delay and gap increase signals are among the more prevalent types of signals relayed from FEF to SC. Furthermore, we found that fixation-related signals in FEF do not go preferentially to the rostral SC. Rostral biases, however, were found in the projections of neurons carrying other types of foveal-related signals, i.e., gap decrease signals and foveal visual signals. We will discuss each of these findings in turn.

It has long been hypothesized that processes occur in the brain (which we call cognitive operations) that are not directly observable to psychophysicists but exert considerable influence over the motor behavior of primates. The discovery of delay signals in prefrontal cortex (Fuster and Alexander 1971; Kubota and Niki 1971) provided a clear candidate for a neuronal 
A

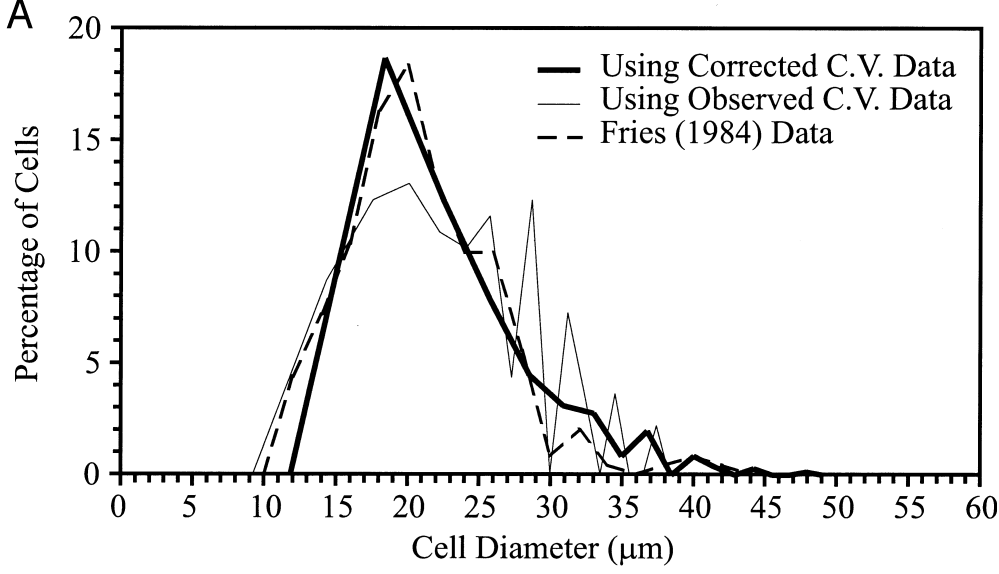

B

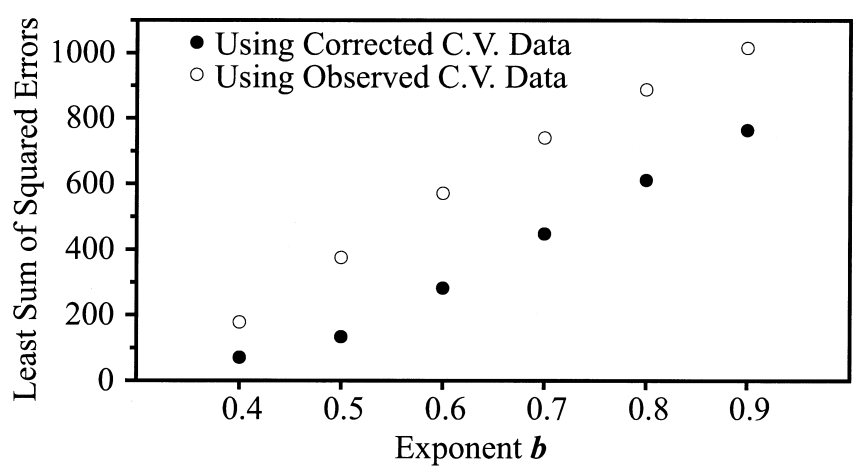

correlate to cognitive operations. Some of these delay signals are spatially selective (Kubota et al. 1974; Niki 1974) and therefore might play a role in spatially restricted attention, memory, or movement planning (Niki and Watanabe 1976). These early experiments studied hand or arm movements. Cognitive operations also influence the generation of eye movements (a brief list of relevant studies and reviews includes Andrews and Coppola 1999; Ballard et al. 1992; Buswell 1935; Hoffman and Subramaniam 1995; Kowler 1990; Land and Furneaux 1997; Miyashita et al. 1996; Paré and Munoz 1996; Sommer 1997; Yarbus 1967), and studies also have found spatially selective delay signals in prefrontal cortex, including within the FEF, that are related to eye movements (Bruce and Goldberg 1985; Funahashi et al. 1989; Joseph and Barone 1987).

Although there have been reports that delay signals exist in the FEF and that these signals may be related to cognitive operations such as spatial memory (Funahashi et al. 1989) or deciding which saccade to make (Kim and Shadlen 1999), where these FEF delay signals go has been entirely unknown. We demonstrated here for the first time that at least some of them go to the SC. Delay signals from FEF therefore may be a major source of the delay signals expressed by SC neurons. The relay of delay signals from FEF to SC may help to strengthen an animal's cognitive influence over its oculomotor behavior, because compared with the FEF, the SC has more direct access to the reticular formation premotor neurons that synapse directly onto muscle-innervating neurons (reviewed by Büttner-Ennever and Büttner 1988). Therefore delay signals in the $\mathrm{SC}$, compared with those in FEF, are in better position to
FIG. 14. Test of whether the conduction velocity correction procedure succeeded in accounting for some of the sampling bias due to cell size variation. We compared the actual distribution of soma diameters of FEF neurons (Fries 1984) with estimated distributions derived from corrected or experimentally observed conduction velocity (C.V.) distributions using the relation Diameter $=a(\mathrm{C} . \mathrm{V} .)^{b}$. $A$ : overall best fits of the estimated soma diameter distributions compared with the actual distribution of Fries (1984). The distribution estimated from the corrected conduction velocity distribution provided a superior fit, suggesting that the correction procedure did account for some of the sampling bias. For both estimated curves, exponent $b=0.4$. Coefficient $a$ was 8.2 for the corrected-data curve and 6.4 for the observed-data curve. $B$ : for all values of the exponent $b$ from 0.4 to 0.9 , the least sum of squared errors between estimated and actual cell diameter distributions was smaller when the estimated distribution was derived using the corrected, rather than the observed, conduction velocity data. Squared errors were measured at $1-\mu \mathrm{m}$ increments along the cell diameter abscissa. increase the excitability of select groups of premotor neurons through tonic depolarization (see Kojima et al. 1996).

We do not mean to imply that FEF delay signals go only to $\mathrm{SC}$, or that the $\mathrm{SC}$ receives delay signals only from the FEF. Further work is needed to identify other recipients of FEF delay signals. The $\mathrm{SC}$ receives delay signals also from the substantia nigra (Hikosaka and Wurtz 1983) and the lateral intraparietal area (Paré and Wurtz 1997).

Gap increase signals in the FEF are similar to delay signals in that both classes of signals may reflect cognitive operations and both are often carried by the same neurons. However, they are different in that gap increase signals seem to be less spatially selective. FEF gap increase signals therefore may primarily reflect nonspatial cognitive operations such as fixation disengagement (Dias and Bruce 1994). It was suggested by Dias and Bruce (1994) that the nonspatial effects of FEF neurons carrying gap increase signals may be mediated in part by their uniform pattern of projection onto the SC. In the present study we confirmed part of this hypothesis, in that we found that many FEF neurons that carry gap increase signals do in fact project to the SC. Furthermore, our EPI results were consistent with the idea that these neurons project uniformly across the SC.

Twenty of our neurons had fixation-related signals, and these projected about equally to rostral and caudal SC, exhibiting no bias in their EPIs (regarding the 2 that were Pure Fixation Neurons, one had an EPI of -1.0 and the other of 1.0). This suggests that the primary purpose of FEF fixationrelated corticotectal neurons is not to drive rostral SC fixation neurons (Munoz and Wurtz 1993a). Rather, they might work in 
parallel to rostral SC fixation neurons, causing inhibition of saccade-related neurons throughout the SC (Munoz and Istvan 1998). The eight neurons that we found with omnispatial movement fields and carrying postsaccadic signals also may contribute to this function.

The above result concerning fixation-related signals is consistent with the comment by Segraves and Goldberg (1987, their p. 1399) that FEF "foveal neurons were antidromically excited from a wide range of points within the topographic representation in the superior colliculus." However, we also found two types of FEF neurons with foveal-related signals that did appear to project in a biased manner to the SC, being antidromically activated more easily from the rostral than the caudal SC. The first type, neurons carrying gap decrease signals, had four characteristics in common with rostral SC neurons: decreased activity during the gap (Dorris and Munoz 1995) and often foveal visual responses, presaccadic drops in activity, and postsaccadic increases in activity (Munoz and Wurtz 1993a). The second type, the relatively rare Pure Foveal Visual Neurons, had foveal visual responses in common with rostral SC neurons. We conclude that in these two types of rostral-projecting FEF neurons we might be seeing signals that converge on rostral SC neurons, contributing at least partially to their activity profiles.

The paucity of Pure Fixation Neurons in our sample was surprising to us, and it leads to the question of whether the FEF was sufficiently explored. We did investigate a large portion of the FEF. The overall cortical area sampled (Fig. 8) was in accord with prior estimates of the range and shape of the FEF and its layer V. The shape (approximately an inverted triangle) is consistent with that described for layer $\mathrm{V}$ by Stanton et al. (1989), who noted that FEF layer V was about twice as wide mediolaterally at the lip compared with at the fundus. The mediolateral range of our corticotectal neurons, $\sim 6.2 \mathrm{~mm}$ (Fig. 8 ), covered much of the 10-mm range estimated for FEF in general (Bruce et al. 1985) and for layer V specifically (Stanton et al. 1989). We explored medially and laterally beyond this range, but none of the corticotectal neurons we encountered were saccade-related (and hence these penetrations were considered outside the FEF), and none were Pure Fixation Neurons. The dorsoventral range of our corticotectal neurons, $\sim 10$ mm (Fig. 8), fully covered the dorsoventral extent as illustrated in prior studies of the FEF or its layer V (Bruce et al. 1985, their Fig. 9, $8 \mathrm{~mm}$; Segraves and Goldberg 1987, their Figs. 5 and 7, $7 \mathrm{~mm}$; Stanton et al. 1989, their Fig. 1, $\sim 6 \mathrm{~mm})$. The estimated depths of our cell body locations probably were somewhat exaggerated as an artifact of the electrode dimpling the cortex during penetrations.

\section{Gradient of visual and movement field eccentricity in the FEF that projects onto the SC}

We found a significant gradient of visual and movement field eccentricity in the FEF that was oriented $\sim 19^{\circ}$ up relative to the arcuate lip (Fig. 11, $A-C$ ). This demonstrates explicitly for the first time that an eccentricity map exists in a specific population of neurons in the FEF, in this case the corticotectal population concentrated in layer $\mathrm{V}$. In the future it will be necessary to determine whether eccentricity maps exist in other populations of FEF neurons as well, which may include neurons of other laminae. It is notable that the oblique orientation of the eccentricity gradient of FEF corticotectal neurons may explain why saccades evoked electrically from FEF decrease in amplitude not only from medial to lateral but also from dorsal to ventral (Bruce et al. 1985).

We also found that within the FEF the gradients of eccentricity and of projection to the SC were closely aligned (Fig. $11 C$ ). When the eccentricity map of FEF afferents was superimposed onto the eccentricity map of the SC (Fig. 12), we found good agreement between the two maps. The eccentricity map of FEF afferents appeared to be logarithmic, like the SC map, and both maps were similarly scaled. However, the FEF afferent map was shifted $\sim 1 \mathrm{~mm}$ rostral compared with the SC map. Future histological studies using punctate injection of anterograde tracer within precisely defined regions of the FEF corticotectal map would be helpful in determining whether neurons in the FEF truly do project to regions in SC that represent slightly less eccentric space. Regardless, our critical point is that FEF neurons representing a certain eccentricity project closely to the location in SC that contains neurons representing similar eccentricities. This confirms prior suggestions from anatomic (Komatsu and Suzuki 1985; Stanton et al. 1988) and electrophysiological studies (Schlag-Rey et al. 1992; Segraves and Goldberg 1987) that much of the FEF seems to be hardwired as a function of eccentricity onto the SC.

Our results concerning patterns of projection onto the $\mathrm{SC}$ rely on our method for estimating axonal terminations. It is important to consider whether this method was sound. Although our intent was to electrically stimulate axon terminals, axons of passage also might have been stimulated. We have two reasons to believe that we stimulated terminals much more often than axons of passage. First, most FEF afferents enter at the rostral pole of the SC (Stanton et al. 1988) and proceed caudally, with some terminating along the way. If our electrodes preferentially activated axons of passage, it should have been much easier to activate FEF neurons from the rostral stimulating electrode than from the caudal one. This would have caused our EPI distributions to be strongly skewed toward -1.0 with few, if any, values at 1.0. In fact, however, the EPI distributions tended to be symmetric (Fig. 9C), which is more consistent with the preferential activation of relatively homogeneously distributed elements such as axon terminals. Second, we found a systematic topography of the FEF projection onto the SC that was consistent with prior anatomic studies (Fig. $11 C$ ), i.e., in general the medial FEF projected to caudal SC and the lateral FEF projected to rostral SC (Komatsu and Suzuki 1985; Stanton et al. 1988). This projection topography in our data are easy to explain if axon terminals were preferentially activated but hard to explain if axons of passage were preferentially activated. We conclude therefore that axon terminals were preferentially activated and that we were generally successful at distinguishing rostral from caudal projections in the SC.

We speculate that the preferential activation of axon terminals was due to a higher density of axon terminals than axon main fibers near our stimulating electrode tips, which we always tried to position within the SC's intermediate gray layer. Corticotectal afferents that enter the rostral SC (Stanton et al. 1988) probably course through the intermediate white layer (i.e., stratum album intermediale) before turning sharply and ascending into the intermediate gray to terminate. A similar scheme of innervation was described by Cajal for the SC 
superficial layers in many mammalian species (Swanson and Swanson 1995). It should be noted that other afferents from the FEF might follow a dorsal trajectory from the midbrain tegmentum before terminating in the SC (Leichnetz et al. 1981). For this class of afferents, it would be particularly easy to understand how terminals (proximal to the electrode tips) would be activated preferentially over main fibers (more distal and ventral to the electrode tips).

Although we believe that axon terminals were preferentially activated in our experiments, it is possible that axon main fibers were occasionally activated instead. In fact, the slight rostral shift of the FEF afferent map relative to the intrinsic SC map (Fig. 12) is most likely an artifact of occasionally activating axon main fibers. As noted above, most FEF corticotectal afferents probably enter the rostral pole and proceed caudally, terminating along the way. Assume for the moment that the afferent map of eccentricity and the intrinsic SC map truly are in perfect register. Then, if stimulation at an SC site occasionally activates fibers that terminate more caudally in the SC, the average eccentricity of FEF neurons activated from the site will exceed the average eccentricity of FEF neurons that actually terminate near the site. If this occurs at both SC stimulating electrodes, the end result will be to slightly overestimate the eccentricity represented by FEF afferents at both the rostral and caudal sites. The measured afferent map of eccentricity therefore would seem to be shifted rostrally relative to the intrinsic $\mathrm{SC}$ map, as was seen.

\section{Topographies of corticotectal signals within the FEF}

It is important to emphasize that the cortical region we examined appeared to be a single functional entity (because it contained a single, orderly map of eccentricity) that was involved primarily in saccade generation (because throughout its extent we found saccade-related neurons and evoked saccades at low current threshold). However, within this single, general region known as the FEF we detected three local topographies of corticotectal signals. First, Movement Neurons were biased medially, and second, Visual Neurons were biased laterally. These first two topographic biases in signal content correspond in interesting ways to mediolateral variations in FEF anatomy. Stanton et al. (1988) found that the FEF projection to the SC varies across two collicular dimensions: medial FEF (where the Movement Neurons are) projects caudally and also deeply in the SC, whereas lateral FEF (where the Visual Neurons are) projects more rostrally and more shallowly. This is consistent with the fact that neurons in deeper SC exhibit more saccaderelated activity than do neurons in shallower SC, which, in contrast, exhibit more visual-related activity (reviewed by Sparks and Hartwich-Young 1989). Also, medial and lateral FEF differ with respect to their cortical afferents, in that the lateral FEF (where the Visual Neurons are) receives a denser input from the highly visual inferotemporal cortex (Bullier et al. 1996; Schall et al. 1995). Finally, FEF efferents to posterior cortical areas also tend to follow this lateral-visual and medialmotor pattern: only lateral FEF projects to highly visual areas such as inferotemporal cortex, area MT, and areas V3 and V4, whereas the entire FEF (including medial FEF) projects to the saccade-related area LIP (Stanton et al. 1995). In summary, although the FEF is a single functional entity involved in saccade generation, lateral FEF seems to be more visual in nature and medial FEF more purely motor in nature. The FEF also has been separated mediolaterally in terms of cytoarchitecture: medial FEF was designated area $8 \mathrm{~A}$ and lateral FEF area 45 by Walker (1940), and this basic dichotomy has been confirmed repeatedly (e.g., Bonin and Bailey 1947) and is now generally accepted (reviewed by Schall 1997). Hence a structure-function relationship is implied in which area 8A may correspond to the more motor subfield of FEF and area 45 to the more visual subfield.

An interesting implication of this organization is that, due to the underlying eccentricity map of the FEF (Fig. 11B), lateral FEF is not only more visual than medial FEF, but it also tends to represent less peripheral space. This suggests that lateral FEF plays a special role in generating small saccades in tight coordination with visual analysis, for example during the examination of natural visual scenes (a behavior in which most saccades are $<15^{\circ}$ in amplitude) (e.g., Andrews and Coppola 1999; Bahill et al. 1975; Henn and Cohen 1973).

Finally, the third type of signal topography within the FEF was that neurons carrying signals thought to reflect cognitive operations (delay and gap increase signals) were biased dorsally. This bias is correlated with a prominent gradation in FEF cytoarchitecture: layer IV is thick and densely packed in the dorsal half of the FEF but tapers off ventrally toward the fundus of the arcuate sulcus (e.g., Stanton et al. 1989). Therefore assuming a radial organization of signal flow between layers, the activity of layer IV neurons in FEF might contribute to the delay and gap increase signals of corticotectal neurons in layer V. Moreover, thalamocortical afferents terminate in layer IV and thus might play a role in generating delay and gap increase signals via reentrant circuits (Fuster 1997) perhaps involving the FEF-SC-thalamus-FEF loop (Lynch et al. 1994; Sommer and Wurtz 1998a).

We thank M. E. Goldberg, M. Paré, M. A. Basso, D. P. Hanes, N. L. Port, E. J. Tehovnik, and two anonymous referees for helpful suggestions and M. K. Smith for histological expertise. We are grateful to the Laboratory of Diagnostic Radiology Research at the National Institutes of Health for magnetic resonance images.

Address for reprint requests: M. A. Sommer, Laboratory of Sensorimotor Research, National Eye Institute, National Institutes of Health, Bldg. 49, Rm. 2A50, 9000 Rockville Pike, Bethesda, MD 20892-4435.

Received 11 August 1999; accepted in final form 28 December 1999.

\section{REFERENCES}

Andersen, R. A. And GnADt, J. W. Reviews of Oculomotor Research. The Neurobiology of Saccadic Eye Movements. Amsterdam: Elsevier, 1989, vol. 3, p. 315-335.

Anderson, R. W., Keller, E. L., Gandhi, N. J., And Das, S. Two-dimensional saccade-related population activity in superior colliculus in monkey. J. Neurophysiol. 80: 798-817, 1998.

ANDREWS, T. J. AND COPPOLA, D. M. Idiosyncratic characteristics of saccadic eye movements when viewing different visual environments. Vision Res. 39: 2947-2953, 1999.

Apter, J. A. Projection of the retina on superior colliculus of cats. J. Neurophysiol. 8: 123-134, 1945.

BAhILl, A. T., AdLER, D., AND STARK, L. Most naturally occurring human saccades have magnitudes of 15 degrees or less. Invest. Ophthalmol. Vis. Sci. 14: 468-469, 1975.

Ballard, D. H., Hayhoe, M. M., Li, F., and Whitehead, S. D. Hand-eye coordination during sequential tasks. Philos. Trans. R. Soc. Lond. B Biol. Sci. 337: 331-339, 1992.

Basso, M. A. Cognitive set and oculomotor control. Neuron 21: 665-668, 1998. 
Basso, M. A. AND WuRTZ, R. H. Modulation of neuronal activity in superior colliculus by changes in target probability. J. Neurosci. 18: 7519-7534, 1998.

BızzI, E. Discharge of frontal eye field neurons during saccadic and following eye movements in unanesthetized monkeys. Exp. Brain Res. 6: 69-80, 1968.

von Bonin, G. AND BAILEy, P. The Neocortex of Macaca mulatta. Urbana, IL: The University of Illinois Press, 1947, p. 30.

Bruce, C. J. AND Goldberg, M. E. Primate frontal eye fields. I. Single neurons discharging before saccades. J. Neurophysiol. 53: 603-635, 1985.

Bruce, C. J., Goldberg, M. E., Bushnell, M. C., and Stanton, G. B. Primate frontal eye fields. II. Physiological and anatomical correlates of electrically evoked eye movements. J. Neurophysiol. 54: 714-734, 1985.

Bullier, J., Schall, J. D., AND Morel, A. Functional streams in occipitofrontal connections in the monkey. Behav. Brain Res. 76: 89-97, 1996.

Burman, D. D. And Bruce, C. J. Suppression of task-related saccades by electrical stimulation in the primate's frontal eye field. J. Neurophysiol. 77: 2252-2267, 1997.

Buswell, G. T. How People Look at Pictures. Chicago, IL: Univ. of Chicago Press, 1935, p. 136-141.

BÜtTNER-EnNeVER, J. A. AND BÜtTnER, U. Reviews of Oculomotor Research. Neuroanatomy of the Oculomotor System. Amsterdam: Elsevier,1988, vol. 2, p. 119-176.

Crist, C. F., Yamasaki, D.S.G., Komatsu, H., and Wurtz, R. H. A grid system and a microsyringe for single cell recording. J. Neurosci. Methods 26: $117-122,1988$.

Cullheim, S. Relations between cell body size, axon diameter and axon conduction velocity of cat sciatic $\alpha$-motoneurones. Neurosci. Lett. 8: 17-20, 1978.

Cynader, M. AND Berman, N. Receptive-field organization of monkey superior colliculus. J. Neurophysiol. 35: 187-201, 1972.

Deng, S.-Y., Goldberg, M. E., Segraves, M. A., Ungerleider, L. G., And Mishrin, M. Adaptive Processes in Visual and Oculomotor Systems. Oxford, UK: Pergamon, 1986, p. 201-208.

DiAS, E. C. AND BRUCE, C. J. Physiological correlate of fixation disengagement in the primate's frontal eye field. J. Neurophysiol. 72: 2532-2537, 1994.

Dias, E. C. AND Segraves, M. A. Muscimol-induced inactivation of monkey frontal eye field: effects on visually and memory-guided saccades. J. Neurophysiol. 81: 2191-2214, 1999.

DorRIS, M. C. AND MunOz, D. P. A neural correlate for the gap effect on saccadic reaction times in monkey. J. Neurophysiol. 73: 2558-2562, 1995.

DoRRIS, M. C. AND MunOZ, D. P. Saccadic probability influences motor preparation signals and time to saccadic initiation. J. Neurosci. 18: 70157026, 1998

EIFUKU, S. AND WURTZ, R. H. Response to motion in extrastriate area MSTl: center-surround interactions. J. Neurophysiol. 80: 292-296, 1998.

FRIES, W. Cortical projections to the superior colliculus in the macaque monkey: a retrograde study using horseradish peroxidase. J. Comp. Neurol. 230: 55-76, 1984.

FunAHASHI, S., BRUCE, C. J., AND GoldMAN-RaKic, P. S. Mnemonic coding of visual space in the monkey's dorsolateral prefrontal cortex. J. Neurophysiol. 61: 331-349, 1989

FusteR, J. M. Unit activity in prefrontal cortex during delayed-response performance: neuronal correlates of transient memory. J. Neurophysiol. 36: 61-78, 1973 .

Fuster, J. M. The Prefrontal Cortex. Anatomy, Physiology, and Neuropsychology of the Frontal Lobe (3rd ed.). Philadelphia, PA: Lippincott-Raven, 1997, p. 232, 238-250.

Fuster, J. M. AND AleXANDER, G. E. Neuron activity related to short-term memory. Science 173: 652-654, 1971.

Fuster, J. M., Bauer, R. H., And Jervey, J. P. Cellular discharge in the dorsolateral prefrontal cortex of the monkey in cognitive tasks. Exp. Neurol. 77: 679-694, 1982.

GaLlyas, F. Silver staining of myelin by means of physical development. Neurol. Res. 1: 203-209, 1979.

GaSSER, H. S. The classification of nerve fibers. Ohio J. Sci. 41: 145-159, 1941.

Glimcher, P. W. AND Sparks, D. L. Movement selection in advance of action in the superior colliculus. Nature 355: 542-545, 1992.

GoldBerg, M. E. AND BruCE, C. J. Primate frontal eye fields. III. Maintenance of a spatially accurate saccade signal. J. Neurophysiol. 64: 489-508, 1990.

Goldberg, M. E. And Segraves, M. A. The role of the frontal eye field and its corticotectal projection in the generation of eye movements. Res. Publ. Assoc. Res. Nerv. Ment. Dis. 67: 195-209, 1990.
Guitton, D. AND MandL, G. The effect of frontal eye field stimulation on unit activity in the superior colliculus of the cat. Brain Res. 68: 330-334, 1974.

Hanes, D. P., Patterson, W. F., and Schall, J. D. Role of frontal eye field in countermanding saccades: visual, movement, and fixation activity. J. Neurophysiol. 79: 817-834, 1998.

HANES, D. P. AND WURTZ, R. H. Effect of superior colliculus inactivation on saccades evoked by frontal eye field stimulation. Soc. Neurosci. Abstr. 25: 805, 1999.

HaYs, A. V., Richmond, B. J., AND Optican, B. J. A UNIX-based multiple process system for real-time data acquisition and control. WESCON Conf. Proc. 2: 1-10, 1982.

Henn, V. and Cohen, B. Quantitative analysis of activity in eye muscle motoneurons during saccadic eye movements and positions of fixation. J. Neurophysiol. 36: 115-126, 1973.

HiKosAKA, O. AND WURTZ, R. H. Visual and oculomotor functions of monkey substantia nigra pars reticulata. IV. Relation of substantia nigra to superior colliculus. J. Neurophysiol. 49: 1285-1301, 1983.

HiKOSAKA, O. AND WURTZ, R. H. Modification of saccadic eye movements by GABA-related substances. I. Effect of muscimol and bicuculline in monkey superior colliculus. J. Neurophysiol. 53: 266-291, 1985.

HikosaKa, O. AND WURTZ, R. H. Saccadic eye movements following injection of lidocaine into the superior colliculus. Exp. Brain Res. 61: 531-539, 1986.

Hoffman, J. E. And Subramaniam, B. The role of visual attention in saccadic eye movements. Percept. Psychophys. 57: 787-795, 1995.

Humphrey, D. R. AND CoRrie, W. S. Properties of pyramidal tract neuron system within a functionally defined subregion of primate motor cortex. J. Neurophysiol. 41: 216-242, 1978.

Humphrey, D. R., Corrie, W. S., And Rietz, R. Properties of the pyramidal tract neuron system within the precentral wrist and hand area of primate motor cortex. J. Physiol. Paris 74: 215-226, 1978.

ILG, U. J. AND Hoffman, K.-P. Functional grouping of the cortico-pretectal projection. J. Neurophysiol. 70: 867-869, 1993.

JosePh, J. P. AND BARONE, P. Prefrontal unit activity during a delayed oculomotor task in the monkey. Exp. Brain Res. 67: 460-468, 1987.

JudGe, S. J., RICHMOND, B. J., AND CHU, F. C. Implantation of magnetic search coils for measurement of eye position: an improved method. Vision Res. 20: 535-538, 1980 .

KeRNELl, D. AND ZwaAgStRa, B. Input conductance, axonal conduction velocity and cell size among hindlimb motoneurones of the cat. Brain Res. 204: 311-326, 1981.

Kim, J.-N. AND SHADLEN, M. N. Neural correlates of a decision in the dorsolateral prefrontal cortex of the macaque. Nature Neurosci. 2: 176-185, 1999

Kojima, J., Matsumura, M., Togawa, M., and Hikosaka, O. Tonic activity during visuo-oculomotor behavior in the monkey superior colliculus. Neurosci. Res. 26: 17-28, 1996.

Komatsu, H. AND SuZuKI, H. Projections from the functional subdivisions of the frontal eye field to the superior colliculus in the monkey. Brain Res. 327: 324-327, 1985

KOWLER, E. Reviews of Oculomotor Research. Eye Movements and their Role in Visual and Cognitive Processes. Amsterdam: Elsevier, 1990, vol. 4, p. $1-70$.

Kubota, K., Iwamoto, T., And Suzuki, H. Visuokinetic activities of primate prefrontal neurons during delayed-response performance. J. Neurophysiol. 47: 1197-1212, 1974

Kubota, K. AND Niki, H. Prefrontal cortical unit activity and delayed alternation performance in monkeys. J. Neurophysiol. 34: 337-347, 1971.

LAND, M. F. AND Furneaux, S. The knowledge base of the oculomotor system. Philos. Trans. R. Soc. Lond. B Biol. Sci. 352: 1231-1239, 1997.

Leichnetz, G. R. AND GoldberG, M. E. Reviews of Oculomotor Research. Neuroanatomy of the Oculomotor System. Amsterdam: Elsevier, 1988, vol. 2, p. 365-429.

Leichnetz, G. R., Spencer, R. F., Hardy, S.G.P., and Astruc, J. The prefrontal corticotectal projection in the monkey: an anterograde and retrograde horseradish peroxidase study. Neuroscience 6: 1023-1041, 1981.

LEMON, R. Methods for Neuronal Recording in Conscious Animals. IBRO Handbook Series: Methods in the Neurosciences. New York: Wiley, 1984, vol. 4, p. 96-103.

Lynch, J. C., Hoover, J. E., AND STRICK, P. L. Input to the primate frontal eye field from the substantia nigra, superior colliculus, and dentate nucleus demonstrated by transneuronal transport. Exp. Brain Res. 100: 181-186, 1994. 
MILLER, J. Discrete and continuous models of human information processing: theoretical distinctions and empirical results. Acta Psychol. (Amst.) 67: 191-257, 1988

Miyashita, K., Rank, M. K., Miyachi, S., and Hikosaka, O. Anticipatory saccades in sequential procedural learning in monkeys. J. Neurophysiol. 76: 1361-1366, 1996.

Movshon, J. A. And Newsome, W. T. Visual response properties of striate cortical neurons projecting to area MT in macaque monkeys. J. Neurosci. 16: 7733-7741, 1996.

MunOz, D. P. AND Istvan, P. J. Lateral inhibitory interactions in the intermediate layers of the monkey superior colliculus. J. Neurophysiol. 79: 11931209, 1998.

MunOz, D. P. AND WurTz, R. H. Fixation cells in monkey superior colliculus I. Characteristics of cell discharge. J. Neurophysiol. 70: 559-575, 1993a.

Munoz, D. P. AND Wurtz, R. H. Fixation cells in monkey superior colliculus. II. Reversible activation and inactivation. J. Neurophysiol. 70: 576-589, 1993 b.

MunOz, D. P. AND WuRTz, R. H. Saccade-related activity in monkey superior colliculus. I. Characteristics of burst and buildup cells. J. Neurophysiol. 73: 2313-2333, 1995.

NIKI, H. Differential activity of prefrontal units during right and left delayed response trials. Brain Res. 70: 346-349, 1974.

Niki, H. AND Watanabe, M. Prefrontal unit activity and delayed response: relation to cue location versus direction of response. Brain Res. 105: 79-88, 1976.

OtTES, F. P., VAN GisBergen, J.A.M., AND EGGERMONT, J. J. Visuomotor fields of the superior colliculus: a quantitative model. Vision Res. 26: 857-873, 1986.

Paré, M. AND MunOz, D. P. Saccadic reaction time in the monkey: advanced preparation of oculomotor programs is primarily responsible for express saccade occurrence. J. Neurophysiol. 76: 3666-3681, 1996.

PARÉ, M. AND Wurtz, R. H. Monkey posterior parietal cortex neurons antidromically activated from superior colliculus. J. Neurophysiol. 78: 3493 3497, 1997.

Porter, R. AND Lemon, R. Corticospinal Function and Voluntary Movement. Monographs of the Physiological Society, Number 45. Oxford, UK: Clarendon, 1993, p. 210-246.

Reuter-Lorenz, P. A., Oonk, H. M., Barnes, L. L., And Hughes, H. C. Effects of warning signals and fixation point offsets on the latencies of proversus antisaccades: implications for an interpretation of the gap effect. Exp. Brain Res. 103: 287-293, 1995.

Robinson, D. A. A method of measuring eye movement using a scleral search coil in a magnetic field. IEEE Trans. Biomed. Electr. 10: 137-145, 1963.

Robinson, D. A. Eye movements evoked by collicular stimulation in the alert monkey. Vision Res. 12: 1795-1808, 1972.

Robinson, D. A. AND Fuchs, A. F. Eye movements evoked by stimulation of frontal eye fields. J. Neurophysiol. 32: 637-648, 1969.

SAsLow, M. G. Effects of components of displacement-step stimuli upon latency for saccadic eye movement. J. Opt. Soc. Am. 57: 1024-1029, 1967.

ScHALl, J. D. Neuronal activity related to visually guided saccades in the frontal eye fields of rhesus monkeys: comparison with supplementary eye fields. J. Neurophysiol. 66: 559-579, 1991.

Schall, J. D. Cerebral Cortex. New York: Plenum, 1997, vol. 12, p. 529-530.

Schall, J. D., Morel, A., King, D. J., ANd Bullier, J. Topography of visual cortex connections with frontal eye field in macaque: convergence and segregation of processing streams. J. Neurosci. 15: 4464-4487, 1995.

Schiller, P. H. AND KoERnER, F. Discharge characteristics of single units in superior colliculus of the alert rhesus monkey. J. Neurophysiol. 34: 920936, 1971.

Schiller, P. H., Sandell, J. H., And Maunsell, J.H.R. The effect of frontal eye field and superior colliculus lesions on saccadic latencies in the rhesus monkey. J. Neurophysiol. 57: 1033-1049, 1987.

SCHILler, P. H. AND STRYKeR, M. Single-unit recording and stimulation in superior colliculus of the alert rhesus monkey. J. Neurophysiol. 35: 915924, 1972.

Schiller, P. H., True, S. D., And Conway, J. L. Deficits in eye movements following frontal eye-field and superior colliculus ablations. J. Neurophysiol. 44: 1175-1189, 1980.

Schlag-Rey, M., Schlag, J., And Dassonville, P. How the frontal eye field can impose a saccade goal on superior colliculus neurons. J. Neurophysiol. 67: 1003-1005, 1992.
Schwartz, E. L. Cerebral Cortex. New York: Plenum, 1994, vol. 10, p. 375-395.

Segraves, M. A. And GoldBerg, M. E. Functional properties of corticotectal neurons in the monkey's frontal eye field. J. Neurophysiol. 58: 1387-1419, 1987.

Sommer, M. A. The spatial relationship between scanning saccades and express saccades. Vision Res. 37: 2745-2756, 1997.

Sommer, M. A., Paré, M., And Wurtz, R. H. Instructional dependence of preparatory discharges of superior colliculus neurons. Soc. Neurosci. Abstr. 23: 843, 1997.

Sommer, M. A. And TeHovnik, E. J. Reversible inactivation of macaque frontal eye field. Exp. Brain Res. 116: 229-249, 1997.

Sommer, M. A. AND Wurtz, R. H. Frontal eye field neurons orthodromically activated from the superior colliculus. J. Neurophysiol. 80: 3331-3333, $1998 \mathrm{a}$

Sommer, M. A. And Wurtz, R. H. Properties of macaque frontal eye field neurons driven orthodromically from the superior colliculus. Soc. Neurosci. Abstr. 24: 522, 1998b.

SOMmer, M. A. AND WuRTZ, R. H. The topographic organization of signals sent from frontal eye field to superior colliculus. Soc. Neurosci. Abstr. 25: 805, 1999.

Sparks, D. L. AND Hartwich-Young, R. Reviews of Oculomotor Research. The Neurobiology of Saccadic Eye Movements. Amsterdam: Elsevier, 1989, vol. 3, p. 213-255.

Sparks, D. L., Holland, R., And Guthrie, B. L. Size and distribution of movement fields in the monkey superior colliculus. Brain Res. 113: 21-34, 1976.

Stanton, G. B., Bruce, C. J., And Goldberg, M. E. Topography of projections to posterior cortical areas from the macaque frontal eye fields. J. Comp. Neurol. 353: 291-305, 1995.

Stanton, G. B., Deng, S.-Y., Goldberg, M. E., and McMullen, N. T. Cytoarchitectural characteristic of the frontal eye fields in macaque monkeys. J. Comp. Neurol. 282: 415-427, 1989.

Stanton, G. B., Goldberg, M. E., And Bruce, C. J. Frontal eye field efferents in the macaque monkey. II. Topography of terminal fields in midbrain and pons. J. Comp. Neurol. 271: 493-506, 1988.

Suzuki, H., Azuma, M., And YumiYa, H. Stimulus and behavioral factors contributing to the activation of monkey prefrontal neurons during gazing. Jpn. J. Physiol. 29: 471-489, 1979.

Swanson, N. AND Swanson, L. W. English translation of the French edition (by Azoulay, L.) of Ramón Y Cajal, S., Histology of the Nervous System of Man and Vertebrates, vol. 2. New York: Oxford Univ. Press, 1995, p. $143-157$.

TAYlor, D. A. Stage analysis of reaction time. Psychol. Bull. 83: 161-191, 1976.

TEHOVNIK, E. J. Electrical stimulation of neural tissue to evoke behavioral responses. J. Neurosci. Methods 65: 1-17, 1996.

Towe, A. L. AND Harding, G. W. Extracellular microelectrode sampling bias. Exp. Neurol. 29: 366-381, 1970.

WALKER, A. E. A cytoarchitectural study of the prefrontal area of the macaque monkey. J. Comp. Neurol. 73: 59-86, 1940.

Wallace, M. T., Meredith, M. A., and Stein, B. E. Converging influences from visual, auditory, and somatosensory cortices onto output neurons of the superior colliculus. J. Neurophysiol. 69: 1797-1809, 1993.

Weyand, T. G. AND GAFKA, A. C. Activity of neurons in area 6 of the cat during fixation and eye movements. Vis. Neurosci. 15: 123-140, 1998a

WeyAnd, T. G. AND GAFKA, A. C. Corticostriatal and corticotectal neurons in area 6 of the cat during fixation and eye movements. Vis. Neurosci. 15: 141-151, 1998b.

Wurtz, R. H. AND GoldBERG, M. E. Superior colliculus cell responses related to eye movements in awake monkeys. Science 171: 82-84, 1971.

Wurtz, R. H. AND Sommer, M. A. Instructional dependence of delay activity in the projection from frontal eye field to superior colliculus. Soc. Neurosci. Abstr. 24: 1146, 1998

Yarbus, A. L. Eye Movements and Vision. New York: Plenum, 1967, p. $171-196$. 